\title{
15 Chancen vergangener Fehler: Flächenpotentiale am Weg zu einem automatisierten Mobilitätssystem
}

Mathias Mitteregger, Aggelos Soteropoulos

1. Einleitung

2. Am Weg zu einem automatisierten, vernetzten und serviceorientierten Verkehrssystem

2.1. Flächeninanspruchnahme von Verkehrsträgern

2.2. Flächeninanspruchnahme, Komponenten und Charakteristika unterschiedlicher Verkehrsträger

2.3. Flächeninanspruchnahme des öffentlichen Personennahverkehrs

2.4. Flächeninanspruchnahme des Individualverkehrs

2.5. Die Standorte der neuen Fahrdienstleister

3. Zur Untersuchung Pkw-assoziierter Konversionsflächen in der Stadtregion Wien

3.1. Das Beispiel Stadtregion Wien

3.2. Auswahl der Pkw-assoziierten Betriebe

3.3. Zur Untersuchung auf Stadtregionsebene

4. Ergebnisse der Untersuchung Pkw-assoziierter Konversionsflächen in der Stadtregion Wien

4.1. Untersuchung auf Stadtregionsebene

4.2. Vertiefte Untersuchung in einer Beispielgemeinde

5. Conclusio und Diskussion

Literatur

Mathias Mitteregger

TU Wien, future.lab Research Center

mathias.mitteregger@tuwien.ac.at

Aggelos Soteropoulos

TU Wien, future.lab Research Center \& Forschungsbereich Verkehrssystemplanung (IVS)

aggelos.soteropoulos@tuwien.ac.at 


\section{EINLEITUNG}

Die technologische Entwicklung automatisierter und vernetzter Fahrzeuge wird in jüngerer Zeit deutlich zurückhaltender bewertet als in den frühen Jahren des Hypes. Ein revolutionäres Szenario, in dem es einem Marktakteur aus der IT-Industrie gelingt, abrupt ein automatisiertes Fahrsystem zu lancieren, das in allen Fahrsituationen besteht, die auch menschliche Fahrerlnnen meistern (Beiker 2015), wird mittelfristig weitestgehend ausgeschlossen. Anstelle der angenommenen Revolution steht heute die Herausforderung einer Jahrzehnte dauernden Übergangszeit, des „Langen Level 4“, währenddessen nur Teile des Straßennetzes automatisiert befahren werden können (Mitteregger et al. 2020, Soteropoulos et al. 2020).

Eine umfassende urbane Transformation, mit Folgen weit über den Verkehrssektor hinaus, bleibt trotzdem zu erwarten: Die Vergangenheit hat gezeigt, dass mit dem Wandel von zusammenhängenden technologischen Systemen (vgl. Freeman/Perez 1988), der sich gegenwärtig durch die Automatisierung und Vernetzung des Verkehrs anbahnt bzw. gerade beginnt, Ketten aus Reformen und Revolutionen in Gang gesetzt werden, die Gesellschaften einiges abverlangen und sich auch im gebauten Raum zeigen (vgl. Headrick 1994, Schmitz 2001, Geels/ Schot 2010). Räumliche Effekte des technologischen Wandels unterscheiden sich in Vorreiterregionen in wesentlichen Aspekten zu jenen in den Regionen der „Nachzügler“ (Grübler 1992, Rogers 2003). Eine grundlegende Gemeinsamkeit jedoch bleibt: Während des Übergangs von einem Technologie- bzw. Verkehrssystem zum nächsten schwinden die Flächenbedarfe des alten Systems, während gleichzeitig neue durch das nächste Technologie- bzw. Verkehrssystem entstehen (Grübler 1990). So wurde beispielsweise am Beginn des Bahnzeitalters Platz für die Schieneninfrastruktur und alle möglichen Betriebsflächen geschaffen, indem Gebäude in den Armenvierteln abgerissen (Bruinsma et al. 2008) oder neue Flächen an den Siedlungsrändern ausgewiesen wurden (Bellet 2009, Bertolini/Spit 2005).

Eine entsprechende räumliche Entwicklungsdynamik ist auch am Weg zu einem automatisierten und vernetzten Verkehrssystem zu erwarten. Im vorliegenden Beitrag werden grundlegende Aspekte dieses Wandels systematisch betrachtet und am Fallbeispiel der „functional urban area" (FUA) Wien quantitativ und qualitativ erfasst. In Abschnitt 2 wird der angewandte Analyserahmen dargelegt: Um die direkte Flächeninanspruchnahme unterschiedlicher Verkehrsträger zu erfassen, wird ein Modell vorgestellt, das auf Basis von Produktlebenszyklusanalysen entwickelt wurde. Das Modell wird an den Beispielen des öffentlichen Personennahverkehrs (ÖPNV), des Individualverkehrs und der Fahrdienstleister (wie etwa Uber oder Lyft) illustriert und die jeweils charakteristische Flächeninanspruchnahme der Teilkomponenten der Systeme beschrieben. Am Ende des zweiten Abschnitts werden bereits entstandene Standorte von Fahrdienstleistern, die vielfach als Vorboten automatisierter Mobilitätsservices gesehen werden, detaillierter betrachtet. Auf Basis dieser Analyse von städtebaulichen Merkmalen und Ausstattungskriterien werden allgemeine räumliche Prinzipien für eine mögliche Flächeninanspruchnahme automatisierter Mobilitätsservices entwickelt. In Abschnitt 3 wird das Modell zur Flächeninanspruchnahme von Verkehrssystemen mittels einer GIS-basierten Analyse, d. h. unter Anwendung eines Geoinformationssystems, für die FUA Wien herangezogen. Dazu wurde ein Datensatz von Herold, einem Unternehmen, das auf digitale Medien und Marketingservices spezialisiert ist und die österreichischen Telefonbücher herausgibt, verwendet, in dem Firmen in Österreich nach der wirtschaftlichen Aktivitätsklassifikation, den sogenannten ÖNACE-Kategorien, erfasst und räumlich verortet sind. Betrachtet werden die Komponenten des Systems Individualverkehr, jene des öffentlichen Verkehrs (ÖV) werden explizit nicht betrachtet. In Abschnitt 4 werden die Ergebnisse der Untersuchung Pkw-assoziierter Betriebe hinsichtlich deren Lage und räumlicher Charakteristika dargestellt und so die These des sek- 
toralen Umbaus der Automobilwirtschaft auf den regionalen Maßstab übertragen. Abschnitt 5 schließt unter Berücksichtigung der Ergebnisse der Untersuchung mit einer Conclusio und Diskussion sowie einem Ausblick hinsichtlich der Frage, inwieweit die potentiellen zukünftigen Transformationsflächen im Kontext der Innenentwicklung, die die Entwicklung im Bestand vor jener auf der „grünen Wiese“, auf bisher unbebautem Gebiet, stellt, genutzt werden könnten.

\section{AM WEG ZU EINEM AUTOMATISIERTEN, VERNETZTEN UND SERVICEORIENTIERTEN VERKEHRSSYSTEM}

Automatisierte und vernetzte Mobilität wird meist als Teilmenge der "Shared Mobility“ und „Mobility as a Service" beschrieben bzw. als das Innovationssystem, das eine umfassende Serviceorientierung im Mobilitätssektor durchsetzen könnte (Shaheen/Cohen 2019). Der Transportsektor steht vor einem Wandel, in dessen Verlauf etablierte Gegensatzpaare wie etwa Individual- und öffentlicher Verkehr und auch Personen- und Güterverkehr obsolet werden könnten (Lenz/Fraedrich 2015; Mitteregger et al. 2020: 43). Bislang haben Studien zu möglichen räumlichen Effekten dieses Wandels insbesondere freiwerdende Parkplatzflächen als die Transformationsräume der Zukunft diskutiert (Alessandrini et al. 2015, Heinrichs 2016, Stead/ Vaddadi 2019). Zahlreiche Simulationsstudien zu automatisierten Car- und Ride-Sharing-Services kommen zu dem Ergebnis, dass durch die Abkehr vom Pkw im Privatbesitz der Bedarf an Parkplätzen radikal - um bis zu 80 bis 90 Prozent - sinken kann (Soteropoulos et al. 2019).

Wenn Parkplatzflächen allein als mögliche Transformationsräume diskutiert werden, wird jedoch die Wirkmacht des Wandels von technologischen Systemen unterschätzt. Zum einen ist der Erkenntnishorizont dieser Studien begrenzt, da sich der schwindende Parkplatzbedarf nicht umfassend mit den verwendeten Modellen darstellen lässt und häufig nur ein angenommenes Verhältnis von (Privat-)Fahrzeugen, die durch automatisierte Car- und Ride-Sharing-Services ersetzt werden, zu wegfallenden Parkplätzen herangezogen wird (ebd.). ${ }^{1}$ Dass zum anderen schon der Fokus dieser Studien zu kurz gegriffen ist, zeigt sich daran, wie die wirtschaftlichen Effekte des Wandels im Verkehrssektor bewertet werden, die eben nicht nur die Parkraumbewirtschaftung betreffen. Wenn Mobilität im Besitz zu einer situativen, konsumierten Mobilität wird, dann ist der gesamte Sektor der Automobilwirtschaft (Bormann et al. 2018) und der des öffentlichen Verkehrs betroffen (Sommer 2018) - und alle Betriebe, die diesen beiden Sektoren zugeordnet werden. Die möglichen räumlichen Effekte eines solchen Wandels umfassen somit nicht nur Parkplatzflächen, sondern potentiell alle Flächen, die über Produktion, Vertrieb bis hin zu Wartung und Betrieb unmittelbar mit dem Verkehrssektor verbunden sind.

\subsection{FLÄCHENINANSPRUCHNAHME VON VERKEHRSTRÄGERN}

Um mögliche Transformationsflächenpotentiale umfassend zu berücksichtigen, werden hier alle Wirtschaftszweige betrachtet, die als Komponenten eines zusammenhängenden Systems für das Funktionieren eines Verkehrsträgers unabdingbar sind (Abb. 1). Für die hier angestellte

$1 \quad$ Hier zeigt sich, dass die etablierten Methoden und akzeptierten Analysen für eine Phase der Normalwissenschaft ausgelegt wurden und Paradigmenwechsel nur unzureichend beschreiben (Kuhn 1967). 
Betrachtung (und die gewählte Beispielregion) wird vereinfachend angenommen, dass Firmen, die (Straßen-)Infrastruktur errichten, instandhalten, rückbauen und recyclieren, in geringerem Maße von den Folgen des Wandels betroffen sein werden, da automatisierte und vernetzte Fahrsysteme zumindest Teile der bestehenden Straßeninfrastruktur nutzen können (vgl. unteres Drittel in Abbildung 1 sowie STRIA 2019).

Abbildung 1: Diagrammtische Darstellung des Produktlebenszyklus von Verkehrsträgern

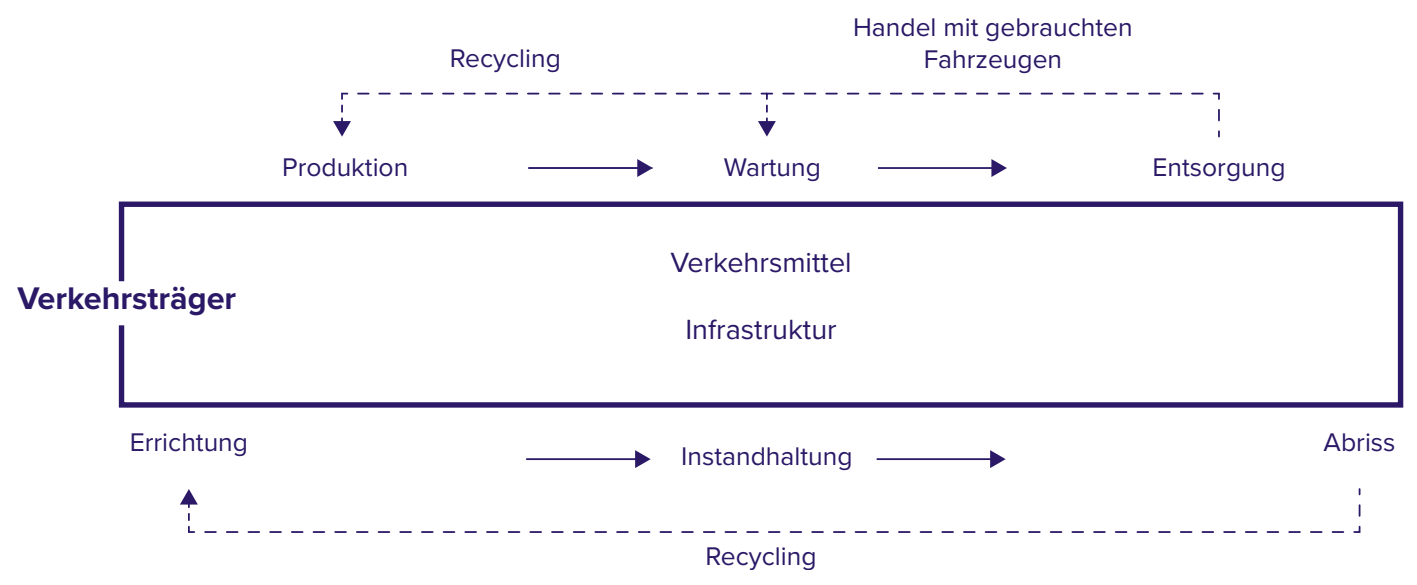

Quelle: eigene Darstellung nach Spielmann/Scholz (2005: 86)

Auf diese allgemeine Systematik aufbauend wurde das in Abbildung 2 dargestellte Modell für die weitere Analyse entwickelt. Neben der Produktion, die in der Beispielregion von marginaler Bedeutung ist, wurde der Handel ergänzt, der vor allem im Individualverkehr von Bedeutung ist. In der Gegenüberstellung der Verkehrsträger Individualverkehr, ÖPNV und Fahrdienstleister werden die die Flächeninanspruchnahme prägenden Charakteristika bereits sichtbar. Fahrdienstleister stehen hier für Firmen wie Uber, Lyft oder Bolt (vgl. Abb. 5 und 6) und auch spezialisierte Drittanbieter wie Splend oder Drover, die Services für FahrerInnen anbieten.

Abbildung 2: Der Produktlebenszyklus ausgewählter Verkehrsträger und derer Komponenten.

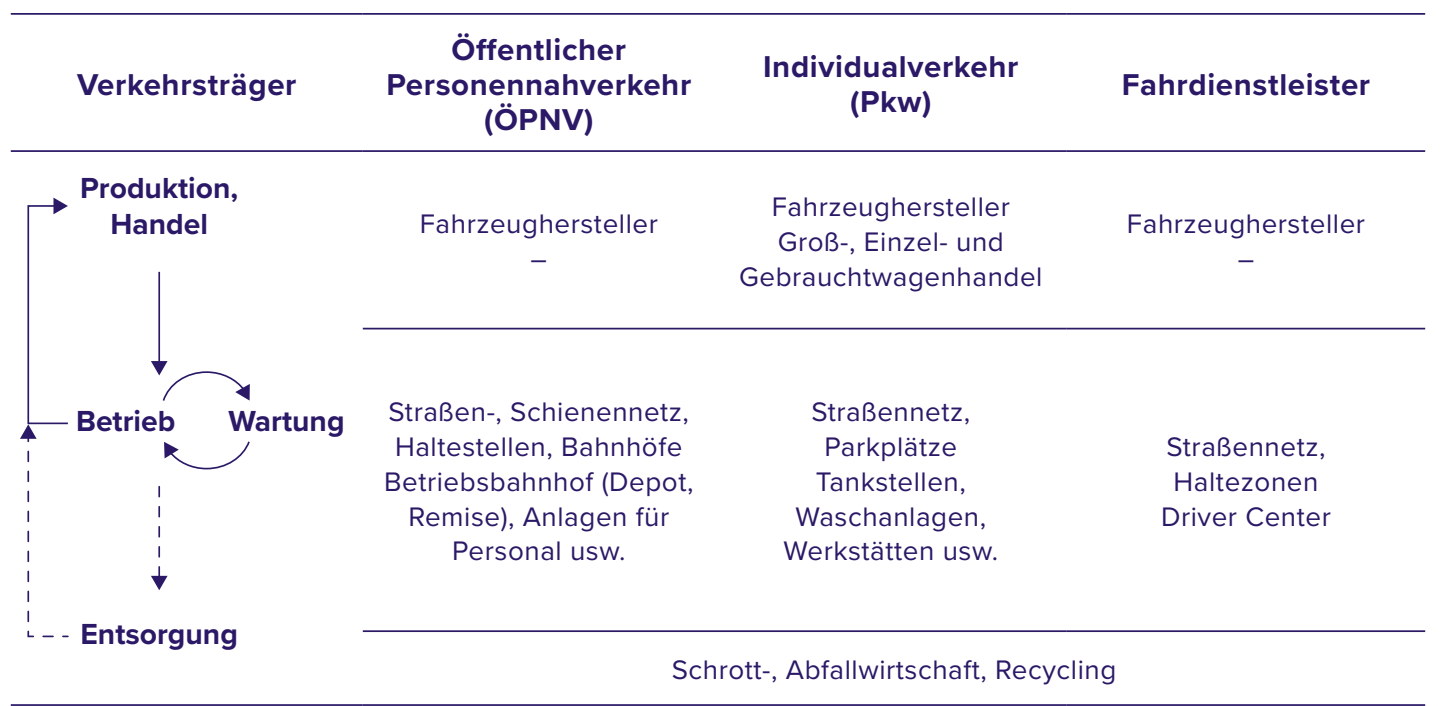


Die Gegenüberstellung der Verkehrsträger in Abbildung 2 zeigt, dass die Komponenten einer serviceorientierten Mobilität, durchgeführt von öffentlichen oder privaten Fahrdienstleistern, andere räumliche Anforderungen stellen werden als jene, die aus dem Individualverkehr bekannt sind: Groß-, Einzel- und Gebrauchtwagenhandel existieren, wenn überhaupt, nur mehr in radikal veränderter Form (vgl. Bormann et al. 2018: 18). Aktuelle Entwicklungen verdeutlichen die Dilemmata, mit denen der Fahrzeughandel in Europa zu kämpfen hat (Eckl-Dorna 2020). Dass auch die regionalen ökonomischen Folgen erheblich sein dürften, zeigt das Mengengerüst des Aftersales-Geschäfts im Individualverkehr (der Handel mit Ersatzteilen, das Durchführen von Reparaturen und Services, technischer Support usw.), dessen Wertschöpfung großteils lokal erbracht wird (Cohen et al. 2006): Die Umsätze in diesem Bereich wurden 2017 für Europa mit 242 Milliarden Euro bewertet (McKinsey 2018) und würden im Zuge der Transformation des Verkehrssystems komplett neu verteilt.

\subsection{FLÄCHENINANSPRUCHNAHME, KOMPONENTEN UND CHARAKTERISTIKA UNTERSCHIEDLICHER VERKEHRSTRÄGER}

Der Blick auf die Vergangenheit macht deutlich, dass mit der Folge von einem dominanten Verkehrsträger zum nächsten Konversionsflächen über alle in Abbildung 2 angeführten Teilbereiche anfielen. Die jeweils unterschiedlichen Entwicklungspotentiale wurden differenziert genutzt: Mit der zunehmenden Durchsetzung des Pkw schrumpfte das Schienennetz und Betriebs- oder Güterbahnhöfe wurden obsolet. Entsprechend schwanden auch Arbeits- und Schlafstätten für das Personal der Eisenbahngesellschaften. Wo Bedarf bestand, wurden all diese Orte zu wertvollen Ressourcen der Siedlungsentwicklung. Anstelle der Güterbahnhöfe und Remisen entstanden in ganz Europa neue Quartiere, urbane Megaprojekte oder wiederum Verkehrsknoten für Hochgeschwindigkeitszüge (Bertolini et al. 2012). Arbeitersiedlungen wurden abgerissen oder adaptiert und neuen Nutzungen zugeführt (vgl. Burghart/Hertenberger 2018). In selteneren Fällen wurden diese Flächen von den Bahnbetreibern nicht immobilienwirtschaftlich verwertet, sondern als Freiräume gesichert. Die räumlichen Charakteristiken der Verkehrsträger werden nachfolgend aus ihrer betrieblichen Logik im Detail entwickelt, wobei besonderes Augenmerk auf die jüngste Gruppe der Fahrdienstleister gelegt wird.

\subsection{FLäCHENINANSPRUCHNAHME DES ÖFFENTLICHEN PERSONENNAHVERKEHRS}

Die Teilkomponenten des ÖPNV sind typischerweise entlang der Infrastruktur konzentriert und in hybriden Betriebsflächen versammelt, die Funktionen wie Wartung, Verkehrsleitung, Parken und andere (Abb. 3). Diese Konzentration rührt vor allem daher, dass lokale Verkehrsbetriebe interne Abläufe möglichst synergetisch und effizient gestalten müssen. Mehrere Funktionen an einem Standort machen es zum Beispiel möglich, allgemeine Flächen im Raumprogramm der Gebäude (Räume für das Personal) oder Nutzungen (z. B. Ersatzteillager für die Garnituren mehrerer Linien) besser auszulasten. Das diese Synergien durchaus über den Verkehrssektor hinausreichen, ist heute noch am Leistungsspektrum kommunaler Infrastrukturdienstleister abzulesen. Die Elektrizität, die in Kraftwerken für Straßen-, U- und S-Bahn gewonnen wird, wird noch heute in städtische Stromnetze eingespeist.

Das Schienennetz und die Betriebsstandorte des ÖPNV bilden, da unzugänglich, meist städtebauliche Barrieren, die mit der Zeit von wachsenden Städten umschlossen wurden. Gleichzeitig macht die hohe Personenfrequenz an Bahnhöfen und Haltestellen diese Standorte attraktiv für andere Funktionen, wie etwa Einzelhandel oder Büros, und hat so die Bildung von Zentren gefördert (Bertolini 2017). 
Abbildung 3: U-Bahn-Bahnhof Erdberg, Wien

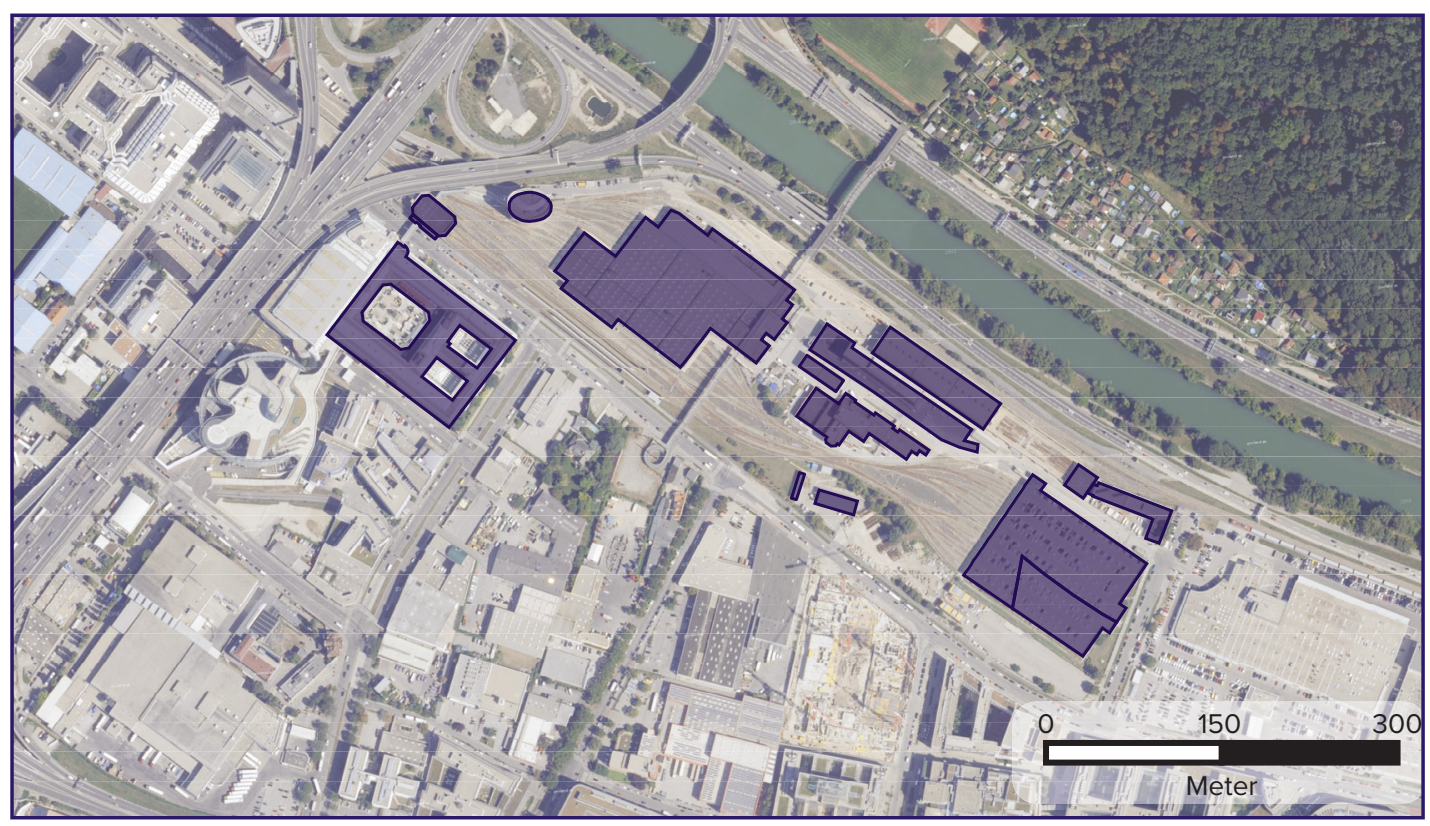

Foto: Vienna GIS

\subsection{FLÄCHENINANSPRUCHNAHME DES INDIVIDUALVERKEHRS}

Der Individualverkehr stellt auf mehreren Ebenen eine Inversion der Prinzipien des ÖPNV dar. Der Pkw im Privatbesitz hat dazu geführt, dass ehemals konzentrierte Funktionen gewissermaBen explodiert sind und nun dispers verteilt entlang des ungleich dichteren Straßennetzes über die gesamte Siedlungsstruktur verteilt liegen. Dadurch hat das Auto dazu beigetragen, dass räumliche Unterschiede nivelliert wurden, eine Gleichförmigkeit, die heute, vor allem wenn vom suburbanen Raum die Rede ist, im Fachdiskurs oft kritisch gesehen wird. Dass dies - zumindest zu Beginn - auch einen sozial-räumlichen Erfolg bedeutete, wird dabei gerne übersehen (McLuhan 2003: 291-301).

Der emotionalen Aufladung des Autos entsprechend, dienen die Komponenten des Individualverkehrs (Autohäuser, Waschstraßen, Werkstätten und Tankstellen) nicht nur der reinen Funktion. Sie sind Ausdruck der Ausdifferenzierung moderner Konsumgesellschaften, da das Auto und dessen Pflege durchaus identitätsstiftend wirken - vor allem dort, wo es keine oder nur unattraktive öffentliche Alternativen gibt (Manderscheid 2014: 606f.). Der mit dem Verkehrsmittel verbundene Stolz wird, anders als bei der Bahn, nicht in Nationen oder Regionen gemeinschaftlich empfunden. Die über das ganze Siedlungsgebiet verteilten Firmenstandorte des Systems Individualverkehr und deren Angebot sind Ausdruck einer fragmentierten mobilen Gesellschaft, deren Mitglieder in der Lage sind, die feinen Unterschiede von Automarken und deren Zubehör zu entschlüsseln (Cresswell 2006, Bourdieu 1987).

Die Pkw-assoziierten Funktionen bleiben im Siedlungsgefüge meist Solitäre bzw. wirken als städtebauliche Barrieren. Dies allerdings weniger aufgrund des eigenen Platzbedarfs der Funktion an sich (der Tankstelle, der Werkstatt oder des Autohauses), sondern in Kombination mit dem exzessiven Flächenverbrauch, der durch den Parkplatzbedarf ausgelöst wird. Im historischen Kontext hat dies den vielleicht stärksten Bruch der Raumentwicklung bisher verantwortet: Das Auto hat den die Stadtgeschichte bestimmenden Zusammenhang von hoher Personenfrequenz und zentralen Orten ins Gegenteil verkehrt. Straßen mit hohen Verkehrsmengen 
können im Zeitalter des Autos nicht länger auch soziale Orte innerhalb des Stadtgefüges sein, wie es zum Beispiel Hauptstraße und Boulevard darstellten (Marshall 2005: 5). Attraktoren wie etwa Einkaufs- oder Fachmarktzentren bedürfen - dieser Logik entsprechend - Autobahnen oder breiter Umfahrungsstraßen, die den Raum durchschneiden, und bleiben durch einen Saum von Parkplatzanlagen selbst doch immer isoliert.

Abbildung 4: Pkw-assoziierte Firmenstandorte entlang der Triester Straße in Wien

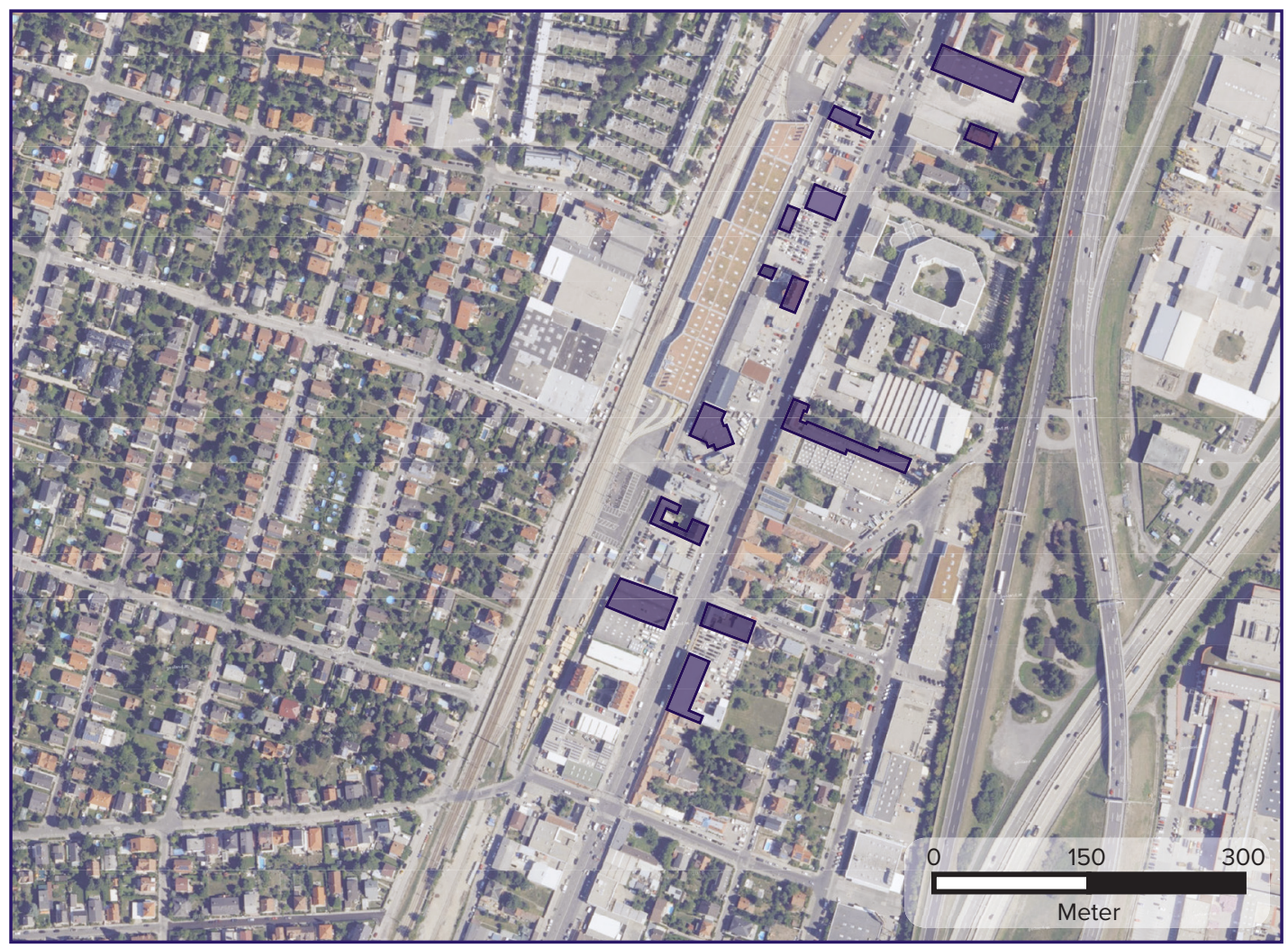

Foto: Vienna GIS

\subsection{DIE STANDORTE DER NEUEN FAHRDIENSTLEISTER}

Die Vorgeschichte der heutigen Fahrdienstleister wie Uber, Lyft oder Bolt ist weniger jene des Taxis, sondern die von Fahrgemeinschaften und Mitfahrzentralen, die Laienfahrerlnnen mit MitfahrerInnen zusammengebracht haben (Chan/Shaheen 2011). Anfangs waren vor allem die persönliche Bekanntschaft oder der gemeinsame Arbeitsplatz Anlass dafür, diese Zweckgemeinschaften zu bilden. Sie entstanden vermehrt zu Zeiten knapper Ressourcen oder/und aus Sparsamkeit (in den USA nach dem 2. Weltkrieg und in Europa verstärkt während der Energiekrise der 1970er Jahre). Daraus entwickelten sich Clubs, Schwarze Bretter und Telefonlisten, die ab den 1990er Jahren verstärkt über das Internet organisiert wurden. Die dafür vorgesehenen Flächen sind, wenn überhaupt, ebenso zweckmäßig angelegt, wie es die Fahrgemeinschaften waren: schlichte Asphaltflächen an Autobahnzubringern, Umfahrungsstraßen oder Rastplätzen.

Während aktuelle Fahrdienstleister dieses auf geteilte Nutzung aufbauende Prinzip im Zuge der Sharing Economy zu einem Geschäftsmodell verkehrten, stellen sich nun auch einige der Effekte unter anderem Vorzeichen dar. Der Erfolg der Fahrdienstleister wirkt bereits auf den 
Verkehrsfluss und den Modal Split (SFCTA 2018), zeigt sich als neuer Platzbedarf an Flughäfen, Bahnhöfen und Einkaufszentren (Edelson 2017, Leiner/Adler 2019) sowie an der Gehsteigkante (vgl. Beitrag 8 von Bruck et al. in diesem Band). Bislang weniger berücksichtigt wurde, dass schon heute ein neuer Gebäudetyp entsteht, der eine Art Hybrid aus Betriebsbahnhof, Rastplatz und Co-Working-Space darstellt (Greenblatt/Shaheen 2015, Epting 2019) und Rückschlüsse auf die mögliche Flächeninanspruchnahme von automatisierten Mobilitätsdienstleistungen geben kann.

Mit der weltweiten Durchsetzung der Fahrdienstleister sind Betriebsstandorte entstanden, in denen entweder der Fahrdienstleister selbst oder spezialisierte Firmen Services für die LaienfahrerInnen und deren Fahrzeuge anbieten. Exemplarisch werden nachstehend vier dieser Standorte und deren Ausstattungsmerkmale verglichen (Abb. 5). In allen untersuchten Fällen scheinen ähnliche Standortqualitäten ausschlaggebend gewesen zu sein, die auch auf die oben skizzierte Historie verweisen. Die Betriebsflächen sind in Stadtrandlage bzw. im suburbanen Siedlungsgebiet an Autobahnknoten gelegen. Damit liegen sie allesamt außerhalb der Gebiete, in denen die meisten Fahrten der Fahrdienstleister nachgefragt werden (Anair et al. 2020). Die Lage an Autobahnabfahrten legt nahe, dass relativ lange Anfahrten (gegenüber geringen Grund- oder Mietpreisen) bewusst in Kauf genommen werden. Bemerkenswert ist deren durchgängig gute Anbindung an den öffentlichen Verkehr. Sie kann darin begründet liegen, dass viele Fahrerlnnen kein eigenes Fahrzeug besitzen, sondern erst einen Wagen beim Fahrdienstleister oder anderen Unternehmen mieten müssen (Zwick 2018).

Abbildung 5: Städtebauliche Merkmale der Betriebsflächen von Fahrdienstleistern

\begin{tabular}{|c|c|c|c|c|}
\hline & Lyft Driver Hub & $\begin{array}{c}\text { Uber Greenlight } \\
\text { Hub }\end{array}$ & $\begin{array}{l}\text { Splend Member } \\
\text { Support Center }\end{array}$ & Bolt Hub \\
\hline Ort & $\begin{array}{l}\text { Windsor Park, } \\
\text { Austin, TX }\end{array}$ & $\begin{array}{c}\text { Bronx, } \\
\text { New York, NY }\end{array}$ & $\begin{array}{l}\text { Cricklewood, } \\
\text { London }\end{array}$ & $\begin{array}{l}\text { Chiswick, } \\
\text { London }\end{array}$ \\
\hline Fläche & $2600 \mathrm{~m}^{2}$ & $2800 \mathrm{~m}^{2}$ & $2300 \mathrm{~m}^{2}$ & k. A. \\
\hline Autobahn & $\checkmark$ & $\checkmark$ & $\checkmark$ & $\checkmark$ \\
\hline Lage & suburban & Stadtrand & Stadtrand & Stadtrand \\
\hline $\begin{array}{l}\text { Öffentlicher } \\
\text { Verkehr }\end{array}$ & Bus & Bus, U-Bahn & Bus, S-Bahn & Bus, S-Bahn \\
\hline
\end{tabular}

Quelle: eigene Darstellung

Die Ausstattungsmerkmale der ausgewählten Betriebsstandorte unterstützen diese Beobachtung (Abb. 6). Hier zeigt sich deutlich, wie wesentliche Angebote für LaienfahrerInnen und Fahrzeuge internalisiert werden. Die angeführten Funktionen wären in der Logik des ÖPNV immanenter Teil des Verkehrsträgers. Bei nach den Prinzipien der Sharing Economy agierenden Fahrdienstleistern sind sie nun Services, die auf Kosten der FahrerInnen zusätzliche Wertschöpfung generieren. Die gängige Praxis, Mitarbeiterlnnen als selbstständige Auftragnehmerlnnen zu sehen (d. h. Laienfahrerlnnen als Unternehmerlnnen, vgl. Heller 2017), erlaubt es, eine neue Mischform zwischen der Logik des ÖPNV und des Individualverkehrs zu erzeugen: Flexible Räume können für das Mietgeschäft, Schulungen, Besprechungen oder CommunityEvents genutzt werden; Einzel-, Verkaufs- oder Beratungsgespräche werden in kleineren Büros mit angehenden oder bestehenden FahrerInnen geführt, wodurch auch dokumentierte Abhängigkeitsverhältnisse entstehen (Abb. 7). 
Abgesehen von Parkflächen, die von den Fahrerlnnen, aber auch für die Mietwagen genutzt werden können, werden zahlreiche Funktionen für den Betrieb und die Wartung der Fahrzeuge angeboten (Abb. 6). Die Arbeit am Fahrzeug wird entweder von den Fahrerlnnen selbst durchgeführt (mit am Standort angebotenem Werkzeug bzw. Garagen) oder von den Betreibern der Standorte (Charpentier 2020): Hier fallen Kosten direkt an oder Mitgliedschaften bilden die Voraussetzung für diesen Service. Um die Auslastung zu erhöhen, werden die angebotenen Services teilweise auch für andere Kundschaft zugänglich gemacht.

Abbildung 6: Ausstattungsmerkmale von Mobilitätsdienstleistungsstandorten

\begin{tabular}{|c|c|c|c|c|}
\hline & $\begin{array}{l}\text { Lyft } \\
\text { Driver Center } \\
\text { (Austin, TX) }\end{array}$ & $\begin{array}{c}\text { Uber } \\
\text { Greenlight Hub } \\
\text { (Bronx New York, NY) }\end{array}$ & $\begin{array}{c}\text { Splend Member } \\
\text { Support Center } \\
\text { (Cricklewood, London) }\end{array}$ & $\begin{array}{c}\text { Bolt Hub } \\
\text { (Chiswick, London) }\end{array}$ \\
\hline $\begin{array}{l}\text { Ruhe- und } \\
\text { Erholungsräume }\end{array}$ & $\checkmark$ & $\checkmark$ & $\checkmark$ & $\checkmark$ \\
\hline Sanitäranlagen & $\checkmark$ & $\checkmark$ & $\checkmark$ & $\checkmark$ \\
\hline $\begin{array}{l}\text { Gastronomie, } \\
\text { Küche, Essbereiche }\end{array}$ & $\checkmark$ & $\checkmark$ & $\checkmark$ & $\checkmark$ \\
\hline Besprechungsräume & $\checkmark$ & $\checkmark$ & $\checkmark$ & $\checkmark$ \\
\hline $\begin{array}{l}\text { Räume für } \\
\text { Veranstaltungen } \\
\text { und Schulungen }\end{array}$ & $\checkmark$ & $\checkmark$ & $\checkmark$ & $\checkmark$ \\
\hline Help Desk & $\checkmark$ & $\checkmark$ & $\checkmark$ & $\checkmark$ \\
\hline $\begin{array}{l}\text { Medizinische } \\
\text { Untersuchungen }\end{array}$ & k. A. & $\checkmark$ & k. A. & k. A. \\
\hline Versicherung & $\checkmark$ & $\checkmark$ & k. A. & k. A. \\
\hline Autovermietung & $\checkmark$ & $\checkmark$ & $\checkmark$ & k. A. \\
\hline Parken & $\checkmark$ & $\checkmark$ & $\checkmark$ & $\checkmark$ \\
\hline $\begin{array}{l}\text { Wartung und } \\
\text { Reparatur }\end{array}$ & $\checkmark$ & $\checkmark$ & $\checkmark$ & k. A. \\
\hline
\end{tabular}

Quelle: Hu (2017), Charpentier (2020), Tucker (2019), Ongweso Jr./Koebler (2019), Splend (2019)

Von den großen Fahrdienstleistern ist bekannt, dass sie sich schon heute auf eine langfristige Übergangszeit hin zu automatisierten Fahrsystemen vorbereiten. Womit gerechnet wird, ist eine andauernde Periode, in der automatisierte Mobilitätsservices und konventionelle, von FahrerInnen erbrachte Dienstleistungen in einem „hybriden Netzwerk“ nebeneinander angeboten werden (Chaum 2019, Sheikh 2018). Das bedeutet, dass bei jeder angefragten Fahrt ein Algorithmus prüft, ob Start und Ziel unter den aktuellen Bedingungen mit einer automatisiert befahrbaren Route verbunden werden können. Trifft dies zu, würde ein hochautomatisiertes Fahrzeug die Fahrt übernehmen (es sei denn, der Kunde schließt dies explizit aus). Wenn dies nicht möglich ist, dann wird die Anfrage an eine/n menschliche/n Fahrerln weitergegeben. Anzunehmen ist, dass die Anzahl der den Fahrerlnnen zuzuordnenden Funktionen schrittweise gegenüber jenen, die den Fahrzeugen zuzuschreiben sind, abnehmen wird. Mögliche neue Nutzungen an diesen Standorten könnten Arbeitsplätze für Disponentlnnen bzw. Personen sein, die ein oder mehrere hochautomatisierte Fahrzeuge überwachen und gegebenenfalls auch steuern (Yankelevich et al. 2018). 
Die Wartung und hier vor allem die Reinigung von hochautomatisierten Fahrzeugen wird als eine äußerst relevante Herausforderung gesehen (Clements/Kockelman 2017: 13; Kucharczyk 2017). Servicebereiche, wie etwa des Lyft Driver Hubs, erinnern an Produktionsstraßen und zeigen, dass auch hier in Zukunft hohe Potentiale für Automatisierung liegen. Firmen, die heute in Nischen (wie etwa der Schwerindustrie und im Bergbau) „predictive maintance“ anbieten, könnten dies auch in Zukunft für Fahrdienstleister tun. Zahlreiche Akteure (Bosch, Intel, Uptake) positionieren sich bereits auf diesem Markt.

Die stadträumliche Wirkung dieser Flächen dürfte sich mit fortschreitender Automatisierung wandeln. Noch ist es der Parkplatzbedarf, der die mangelnde städtebauliche Integration verursacht, weswegen heute diese Betriebsstandorte dem entsprechen, was oben für die Pkw-assoziierten Flächen beschrieben wurde. Mit fortschreitender Automatisierung, währenddessen immer weniger Fahrerlnnen und mehr die Algorithmen steuern, könnten die Parkflächen auch anders genutzt werden: Geparkt würde nur mehr zu Schwachlastzeiten, wobei fraglich ist, ob dies dann nicht auf noch entlegeneren Standorten (wie dies Logistikunternehmen bereits heute tun) passieren könnte, da gegenwärtig bereits beträchtliche Anfahrtszeiten in Kauf genommen werden. Möglich ist, dass andere Funktionen, wie etwa die der Disponentlnnen, global ausgelagert werden. Auch hierfür existieren im Bergbau bereits Anwendungen (Frangoul 2018). Im Straßenraum könnte die Trennwirkung mittelfristig in den Straßen zu und von den Standorten verstärkt werden, wenn eine infrastrukturelle Ertüchtigung für automatisierte Fahrsysteme notwendig würde (Soteropoulos et al. 2020).

Abbildung 7: Werbung spezialisierter Anwälte für Probleme von Ride-Sharing Mitarbeiterlnnen auf einem Bus in Chicago

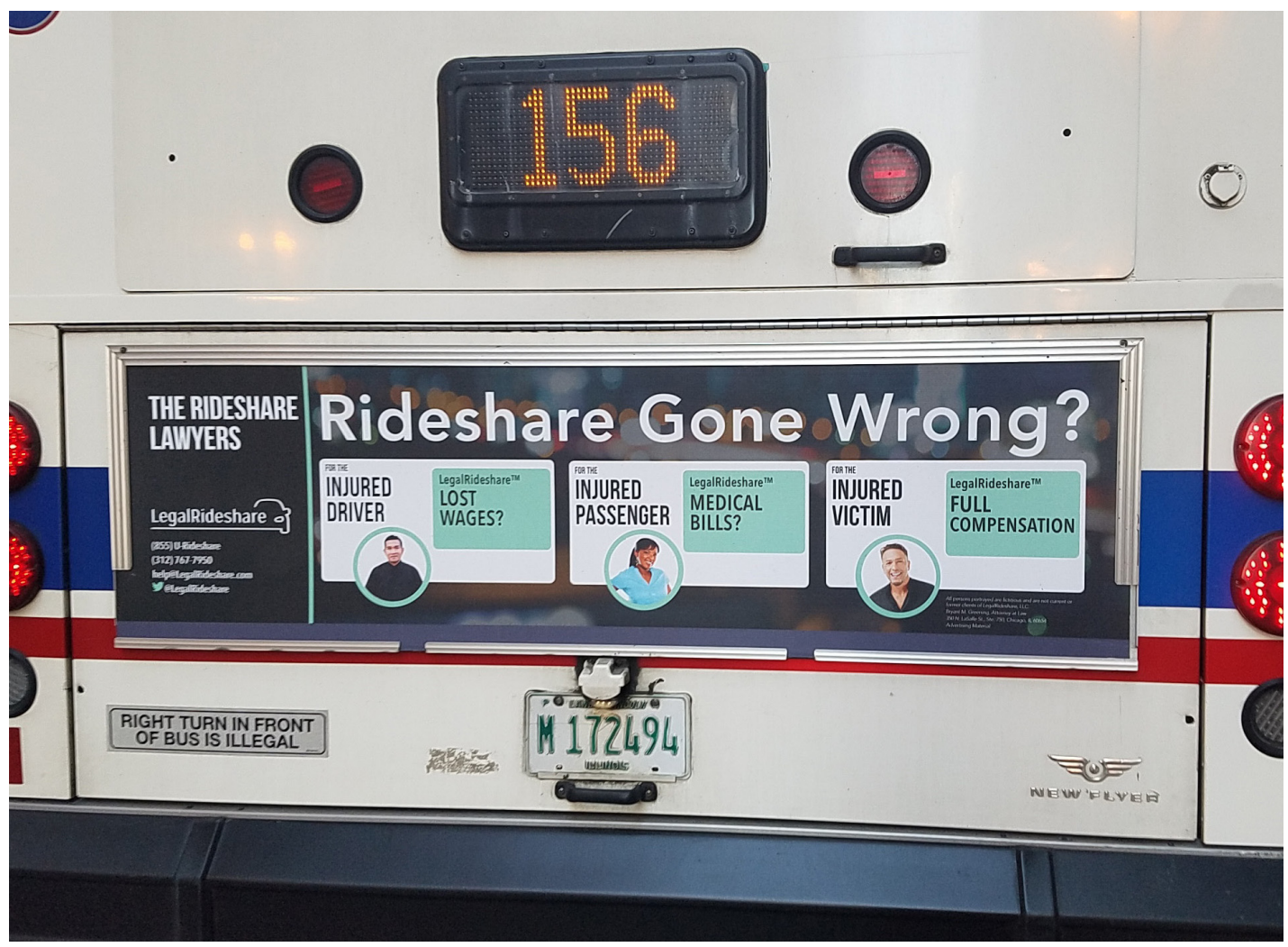

Das hier abgedruckte Bild ist explizit von der Creative Commons Lizenz des Textes ausgenommen. Die Rechte bleiben bei den Verfassern. Foto: Filip Frącz 2017 


\title{
3. ZUR UNTERSUCHUNG PKW-ASSOZIIERTER KONVERSIONS- FLÄCHEN IN DER STADTREGION WIEN
}

\begin{abstract}
Um die Flächenentwicklungsdynamik, die durch die Transformation hin zu einem automatisierten, vernetzten und serviceorientierteren Verkehrssystem ausgelöst werden könnte, im Detail zu untersuchen, wurden am Beispiel der Stadtregion Wien entsprechende Pkw-assoziierte Funktionen und Betriebe erfasst sowie deren Lage und räumliche Charakteristika beschrieben. Die Ermittlung der Pkw-assoziierten Firmen wird mittels des in Abschnitt 2 (Abb. 2) entwickelten Modells durchgeführt. Es geht hier also explizit nur um die Transformation von Bestandsflächen (Steen/Ryding 1993) und nicht um eine mögliche zusätzliche Flächeninanspruchnahme in bisher unbebauten Gebieten (Jolliet/Crettaz 1996). An der Beispielregion der FUA Wien wird illustriert, wo potentielle zukünftige Transformationsflächen liegen und welche Charakteristika diese aufweisen. Abbildung 8 gibt einen Überblick der methodischen Schritte innerhalb der Untersuchung.
\end{abstract}

Abbildung 8: Analyseschritte für die Untersuchung Pkw-assoziierter Konversionsflächen in der Stadtregion

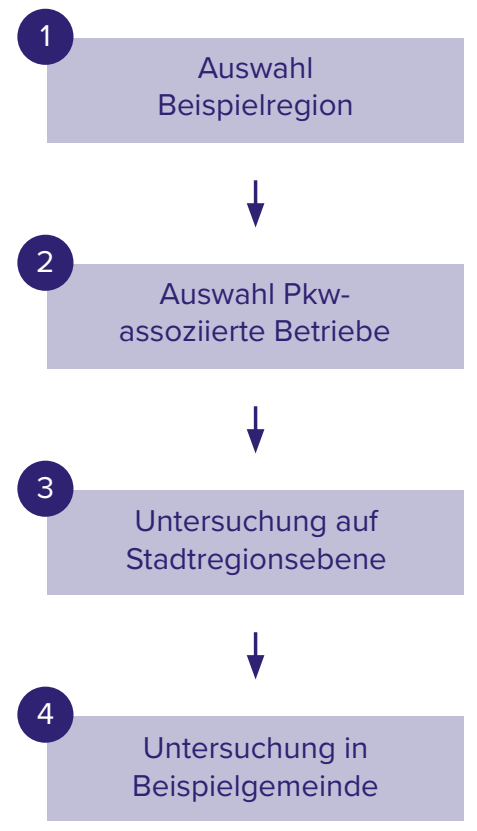

- FUA Wien

ÖNACE-Kategorien

- Produktion und Handel, Betrieb, Wartung

- GIS-gestütze Analyse

- Lage, Flächennutzung, Zentralität

- Differenzierung nach Urban-Rural-Typologie

in

- Räumlich vertiefte Untersuchung

- Auf Grundlage der Untersuchung auf Stadtregionsebene

Quelle: eigene Darstellung

\subsection{DAS BEISPIEL STADTREGION WIEN}

Als Beispielregion für die Untersuchung wurde die FUA Wien, gemäß der Definition und Abgrenzung der OECD (2020), herangezogen. Die FUA Wien umfasst neben der Stadt Wien weitere Gemeinden mit sehr unterschiedlichen räumlichen Charakteristika. Sie weist jedoch hinsichtlich ihrer Siedlungsstruktur und Verflechtung ähnliche Eigenschaften wie andere Stadtregionen im deutschsprachigen Raum auf und eignet sich daher gut als Beispielregion 
für die Untersuchung Pkw-assoziierter Konversionsflächen: Das Aufzeigen von Lage und räumlichen Charakteristika dieser potentiell zukünftigen Transformationsflächen erfolgt beispielhaft in der FUA Wien, ist jedoch auch für andere Stadtregionen relevant bzw. auf diese übertragbar.

Um im Rahmen der Untersuchung eine stärkere Differenzierung vorzunehmen, wurde auf die Urban-Rural-Typologie der Statistik Austria zurückgegriffen (Statistik Austria 2017). Die Stadt Wien, die in dieser Typologie gemeinsam mit einigen Umlandgemeinden in die Kategorie „Urbane Großzentren“ fällt, wurde aufgrund ihrer übergeordneten Bedeutung und der deutlichen Unterschiede gegenüber den Kommunen des Umlands derselben Kategorie eigenständig behandelt. Abbildung 9 gibt einen Überblick der FUA Wien sowie die Unterteilung der Gemeinden gemäß der Urban-Rural-Typologie der Statistik Austria.

Abbildung 9: Beispielregion FUA Wien und Einteilung gemäß Urban-Rural-Typologie der Statistik Austria

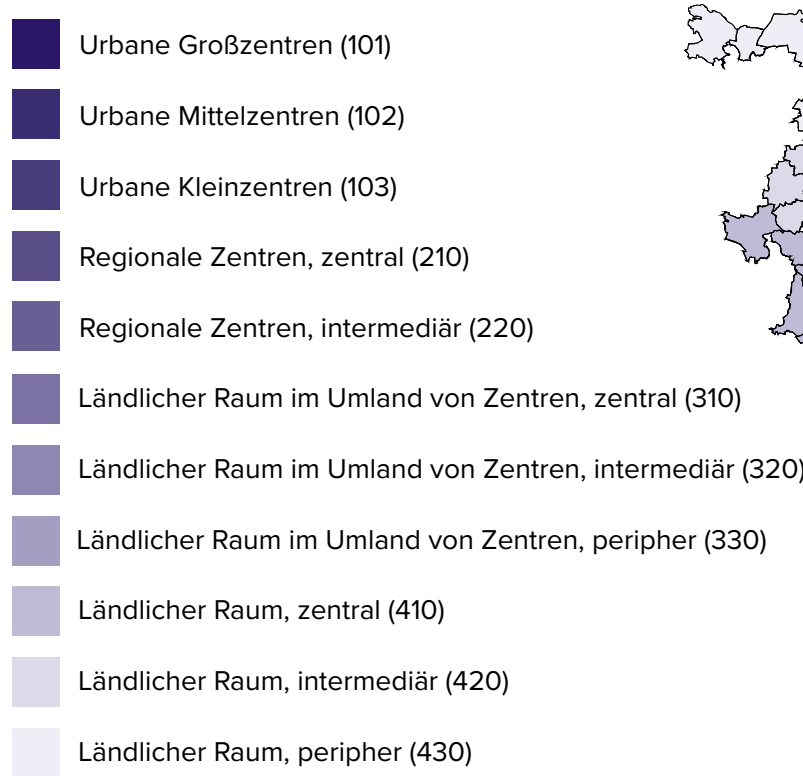

Ländlicher Raum, peripher (430)

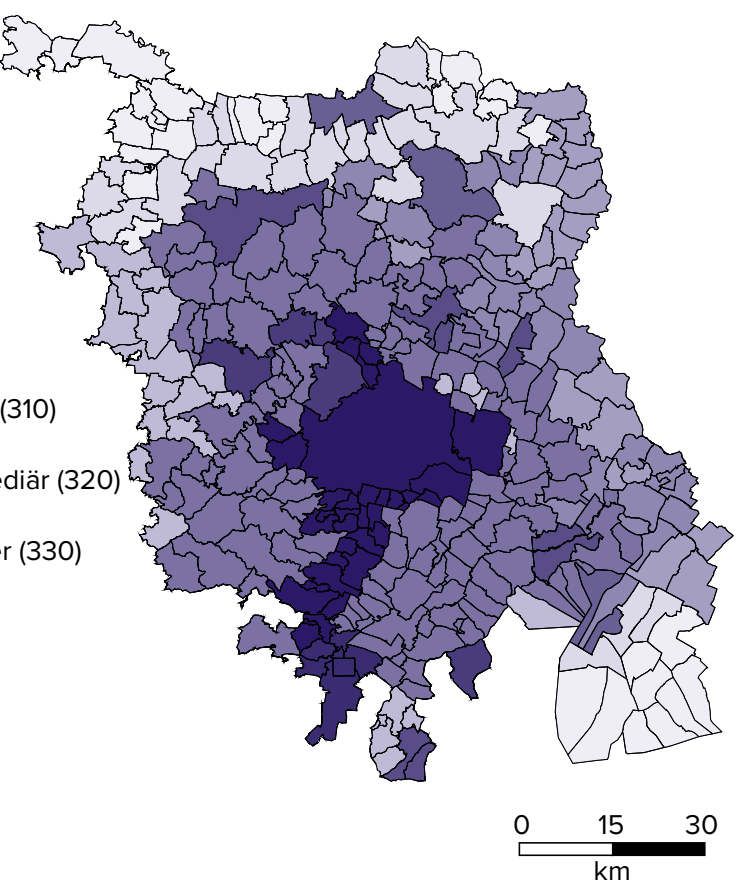

Quelle: eigene Darstellung nach Statistik Austria (2020)

\subsection{AUSWAHL DER PKW-ASSOZIIERTEN BETRIEBE}

Für die Auswahl der Pkw-assoziierten Firmen wurde auf den Datensatz von Herold, eine Datenbank zu Betrieben und Einrichtungen in ganz Österreich, zurückgegriffen. Unter Berücksichtigung der ÖNACE-Kategorien wurden relevante Betriebs- bzw. Einrichtungskategorien identifiziert und gemäß der in Abbildung 2 entwickelten Systematik den Komponenten Produktion/ Handel, Betriebe und Wartung zugeteilt. Jene ÖNACE-Kategorien, die in irgendeiner Form im Zusammenhang mit Teilen bzw. Zubehör von Pkw stehen, wurden der Kategorie Wartung zugeteilt, Betriebe aus dem Bereich der Entsorgung bzw. des Recyclings wurden nicht berücksichtigt. Die herangezogenen ÖNACE-Kategorien sowie die Einteilung in die erläuterte Systematik wird in Abbildung 10 dargestellt. 
Abbildung 10: Auswahl der ÖNACE-Kategorien

\begin{tabular}{|c|c|c|c|c|}
\hline ÖNACE & & $\begin{array}{l}\text { Produktion } \\
\text { und Handel }\end{array}$ & Betrieb & Wartung \\
\hline 29100 & $\begin{array}{l}\text { Herstellung von Kraftwagen und } \\
\text { Kraftwagenmotoren }\end{array}$ & $\checkmark$ & & \\
\hline 29200 & $\begin{array}{l}\text { Herstellung von Karosserien, Aufbauten } \\
\text { und Anhängern }\end{array}$ & $\checkmark$ & & \\
\hline 29310 & $\begin{array}{l}\text { Herstellung elektrischer } \\
\text { und elektronischer } \\
\text { Ausrüstungsgegenstände für } \\
\text { Kraftwagen }\end{array}$ & & & $\checkmark$ \\
\hline 29320 & $\begin{array}{l}\text { Herstellung von sonstigen Teilen und } \\
\text { sonstigem Zubehör für Kraftwagen }\end{array}$ & & & $\checkmark$ \\
\hline 30990 & Herstellung von sonstigen Fahrzeugen & $\checkmark$ & & \\
\hline 33170 & $\begin{array}{l}\text { Instandhaltung und Reparatur von } \\
\text { Kraftwagen }\end{array}$ & & & $\checkmark$ \\
\hline 45111 & $\begin{array}{l}\text { Großhandel mit Kraftwagen mit einem } \\
\text { Gesamtgewicht von 3,5 t oder weniger }\end{array}$ & $\checkmark$ & & \\
\hline 45112 & $\begin{array}{l}\text { Einzelhandel mit Kraftwagen mit einem } \\
\text { Gesamtgewicht von 3,5 t oder weniger }\end{array}$ & $\checkmark$ & & \\
\hline 45200 & $\begin{array}{l}\text { Instandhaltung und Reparatur von } \\
\text { Kraftwagen }\end{array}$ & & & $\checkmark$ \\
\hline 45310 & $\begin{array}{l}\text { Großhandel mit Kraftwagenteilen und } \\
\text {-zubehör }\end{array}$ & & & $\checkmark$ \\
\hline 45320 & $\begin{array}{l}\text { Einzelhandel mit Kraftwagenteilen und } \\
\text {-zubehör }\end{array}$ & & & $\checkmark$ \\
\hline 47300 & $\begin{array}{l}\text { Einzelhandel mit Motorenkraftstoffen } \\
\text { (Tankstellen) }\end{array}$ & & $\checkmark$ & \\
\hline 49320 & Betrieb von Taxis & & $\checkmark$ & \\
\hline 52211 & Parkhäuser und Parkgaragen & & $\checkmark$ & \\
\hline 77111 & $\begin{array}{l}\text { Leasing von Kraftwagen mit einem } \\
\text { Gesamtgewicht von } 3,5 \text { t oder weniger }\end{array}$ & & $\checkmark$ & \\
\hline 77112 & $\begin{array}{l}\text { Vermietung von Kraftwagen mit einem } \\
\text { Gesamtgewicht von 3,5 t oder weniger }\end{array}$ & & $\checkmark$ & \\
\hline 77120 & $\begin{array}{l}\text { Vermietung von Kraftwagen mit einem } \\
\text { Gesamtgewicht von mehr als } 3,5 \mathrm{t}\end{array}$ & & $\checkmark$ & \\
\hline 85530 & Fahr und Flugschulen & & $\checkmark$ & \\
\hline
\end{tabular}

Quelle: eigene Darstellung

\subsection{ZUR UNTERSUCHUNG AUF STADTREGIONSEBENE}

Auf der Stadtregionsebene wurde zunächst die Lage der unterschiedlichen Pkw-assoziierten Funktionen insbesondere im Verhältnis zu sonstigen jeweils dort verorteten Funktionen betrachtet. Zudem wurde untersucht, in welcher Art von Gebieten sich die jeweiligen Betriebe befinden, d. h. welche Flächennutzung diese Gebiete aufweisen. Für diese Auswertung wurden Daten zur Flächennutzung aus OpenStreetMap (einer freien Plattform, die frei nutzbare 
Geodaten sammelt, strukturiert und unter freier Lizenz für die allgemeine Nutzung in einer Datenbank zur Verfügung stellt) herangezogen. Die Auswertung wurde in die Kategorien 1.) Wohngebiet, 2.) Gewerbe-/Industriegebiet, 3.) Gebiet mit Büros/Business Parks, 4.) Gebiet mit Einzelhandelsgeschäften/Einkaufszentren und 5.) andere Gebiete gegliedert (vgl. Ramm 2019: 17; OpenStreetMap Wiki 2020).

Außerdem wurde die Lage der Betriebe zum nächsten Ortszentrum untersucht. Hierbei wurde jeweils die Entfernung der Standorte zum nächsten Gemeindeamt berechnet; die Daten zum Gemeindeamt stammen dabei aus dem Herold-Datensatz. Die Stadt Wien wurde aufgrund ihrer heterogenen Zentrenstruktur bei dieser Analyse ausgeklammert.

Die Untersuchungen zur Lage der Betriebe bzw. zu Funktionen hinsichtlich ihrer Flächennutzung sowie zu deren Entfernung zum nächsten Ortszentrum wurden zudem jeweils gemäß der vorher beschriebenen Urban-Rural-Typologie der Statistik Austria räumlich differenziert

Auf Grundlage der Ergebnisse auf Stadtregionsebene fand zudem eine räumlich vertiefte Untersuchung in einer Beispielgemeinde statt. Hier wurden die Pkw-assoziierten Konversionsflächen hinsichtlich ihrer Eignung für eine Innenentwicklung im Detail untersucht. Ableitend aus den Ergebnissen der Untersuchung auf Stadtregionsebene wurde hierfür Liesing, der 23. Wiener Gemeindebezirk, ausgewählt.

\section{ERGEBNISSE DER UNTERSUCHUNG PKW-ASSOZIIERTER KONVERSIONSFLÄCHEN IN DER STADTREGION WIEN}

\subsection{UNTERSUCHUNG AUF STADTREGIONSEBENE}

\section{Verteilung der Betriebe nach Komponenten des Verkehrssystems und Urban-Rural- \\ Typologie}

In der FUA Wien wurden in Summe 3.082 Standorte von Pkw-assoziierten Betrieben erfasst. Gegliedert nach der Systematik gemäß Abbildung 2 fällt der größte Teil der Betriebe in die Bereiche Wartung (42 \%) sowie Betrieb (40\%). Etwas weniger als ein Fünftel der Pkw-assoziierten Funktionen (18\%) gehört zum Bereich Produktion und Handel (Abb. 11).

Abbildung 11: Verteilung der Pkw-assoziierten Betriebe nach den Bereichen Produktion und Handel, Betrieb und Wartung (in \%)

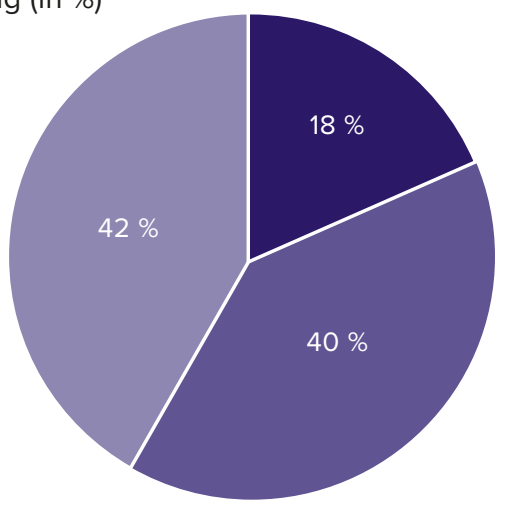

$$
\begin{aligned}
& \text { - Produktion Handel } \\
& \text { - Betrieb } \\
& \text { Wartung }
\end{aligned}
$$


Abbildung 12: Anzahl der Pkw-assoziierten Betriebe nach der Urban-Rural-Typologie ( $n=3.082$ )

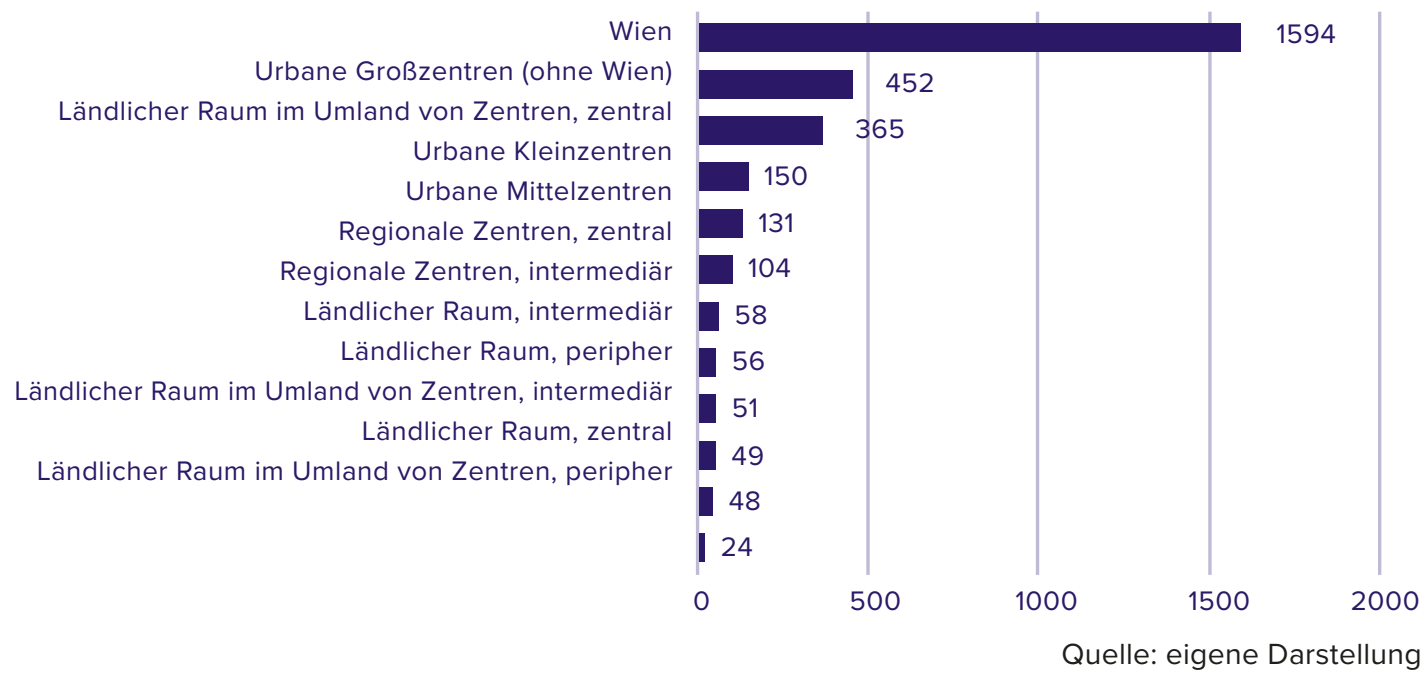

Betrachtet man die Anzahl der Betriebe unterteilt nach der Urban-Rural-Typologie der Statistik Austria (Abb. 12) wird erkennbar, dass sich die größte Anzahl der Pkw-assoziierten Betriebe mit insgesamt 1.594 Betrieben naturgemäß in Wien zeigt, gefolgt von insgesamt 452 Betrieben in den „urbanen Großzentren“ - das Siedlungsband im Süden von Wien - sowie in den Gemeinden im Nordwesten und Südosten entlang der Donau. Insgesamt befinden sich 365 Betriebe in Gemeinden des Typs „Iändlicher Raum im Umland von Zentren, zentral“, die auch flächenmäßig den größten Teil der FUA Wien ausmachen. Verhältnismäßig groß ist die Anzahl der Betriebe in den drei nachstehenden Kategorien „urbane Kleinzentren“ (150 Betriebe), „urbane Mittelzentren“ (131 Betriebe) und „Regionalen Zentren, zentral“ (104 Betriebe), zu welchen die Gemeinden Wolkersdorf, Gänserndorf und Bruck an der Leitha gehören. Letztere sind auch jene Gemeinden mit dem höchsten Anteil von Aus- und Einpendlern unter den Erwerbstätigen am Wohnort (Görgl et al. 2017: 181).

Abbildung 13: Aufteilung der Pkw-assoziierten Betriebe nach den Bereichen Produktion und Handel, Betrieb und Wartung sowie der Urban-Rural-Typologie $(n=3.082)$

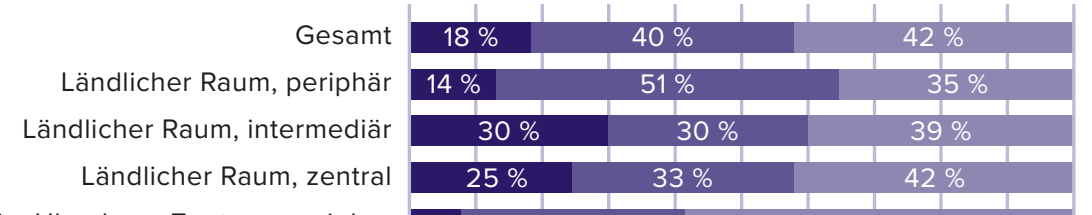

Ländlicher Raum im Uland von Zentren, peripher Ländlicher Raum im Umland von Zentren, intermediär

Ländlicher Raum im Umland von Zentren, zentral Regionale Zentren, intermediär

Regionale Zentren, zentral

Urbane Kleinzentren

Urbane Mittelzentren

Urbane Großzentren (ohne Wien)

Wien

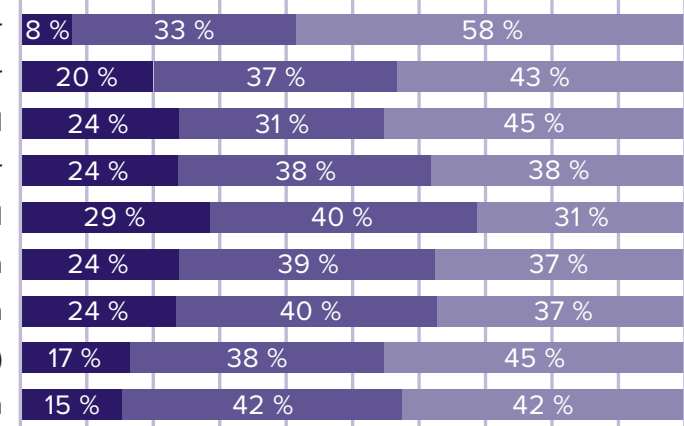

$0 \% 10 \% 20 \% 30 \% \quad 40 \% 50 \% \quad 60 \% \quad 70 \% \quad 80 \% 90 \% \quad 100 \%$

- Produktion Handel

Betrieb Wartung

Quelle: eigene Darstellung 
Die Aufteilung der Betriebe nach den Bereichen Produktion und Handel, Betrieb und Wartung gemäß der Urban-Rural-Typologie zeigt, dass sich hohe Anteile von Betrieben im Bereich Wartung vor allem in der Kategorie "Ländlicher Raum im Umland von Zentren, peripher" (58 \%) und im Bereich Wartung insbesondere in der Kategorie "Ländlicher Raum, peripher" (51 \%) finden. Eine nicht unerhebliche Anzahl von Betrieben im Bereich Produktion und Handel zeigt sich hingegen in bzw. im Umland von Zentren, insbesondere in den regionalen Zentren (29 \%; Abb. 13).

\section{Lage der Betriebe in der Stadtregion}

Abbildung 14 zeigt die Lage der Betriebe in der Stadtregion. Dargestellt wird die Anzahl der Betriebe und deren Anteil im Verhältnis zu an allen im Nahbereich verorteten Betrieben bzw. Einrichtungen. Vor allem im Süden Wiens mehren sich die Orte, die sowohl eine hohe Anzahl

Abbildung 14: Anzahl der Pkw-assoziierten Betriebe und Anteil an allen Betrieben bzw. Einrichtungen in der FUA Wien

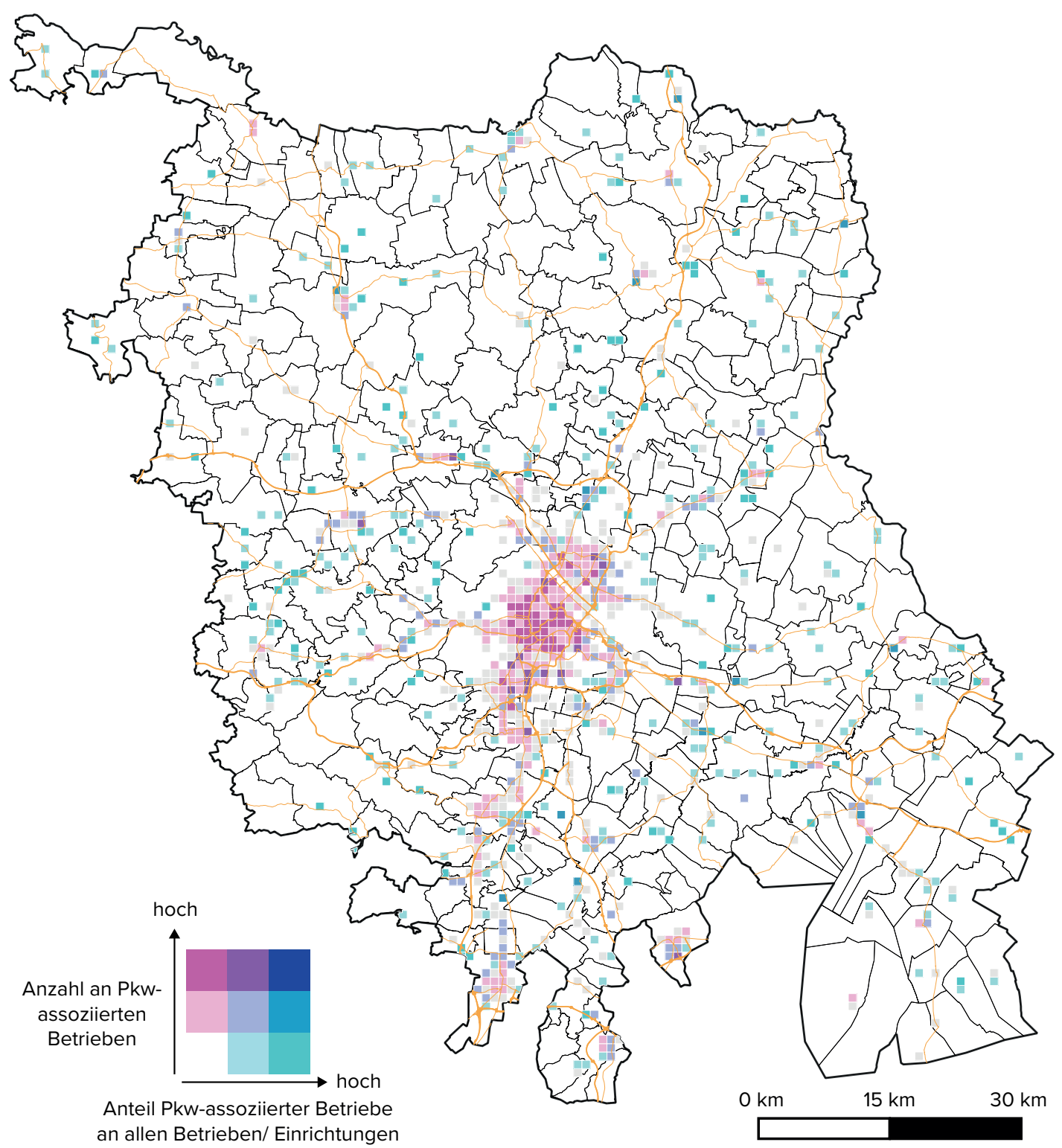

Quelle: eigene Darstellung 
an Pkw-assoziierten Betrieben als auch einen beträchtlichen Anteil dieser Betriebe an allen Betrieben bzw. Einrichtungen aufweisen und somit sehr stark durch diese Betriebe geprägt sind.

\section{Lage der Betriebe nach Flächennutzung}

Hinsichtlich der Lage der Betriebe nach der Flächennutzung (Abb. 15) zeigt sich, dass die Pkwassoziierten Betriebe in der gesamten FUA Wien überwiegend in Wohngebieten (67\%) oder in Gewerbe- bzw. Industriegebieten (14 \%) liegen. Im Vergleich dazu gibt es nur wenige Betriebe in Gebieten mit Büros/Business Parks oder in Gebieten mit Einzelhandelsgeschäften/Einkaufszentren (jeweils $6 \%$ ). In Bezug auf die Urban-Rural-Typologie zeigt sich, dass die Zahl von Pkwassoziierten Betrieben in Gewerbe- bzw. Industriegebieten vor allem in den Zentren, besonders in den urbanen Großzentren (26 \%), sowie im „Ländlichen Raum im Umland von Zentren, zentral“ (17\%) hoch liegt. Hohe Anteile von Betrieben in Wohngebieten zeigen sich hingegen eher in kleineren, peripheren Gemeinden, etwa für die Kategorie „Ländlicher Raum, peripher“ (90 \%) und "Ländlicher Raum im Umland von Zentren“ (88 \%). Interessant ist die hohe Anzahl der Pkw-assoziierten Betriebe in Gebieten mit Einzelhandelsgeschäften bzw. Einkaufszentren in der Kategorie „Regionale Zentren, intermediär“ (29\%).

Abbildung 15: Aufteilung der Pkw-assoziierten Betriebe nach den Bereichen Produktion und Handel, Betrieb und Wartung sowie der Urban-Rural-Typologie $(n=3.082)$

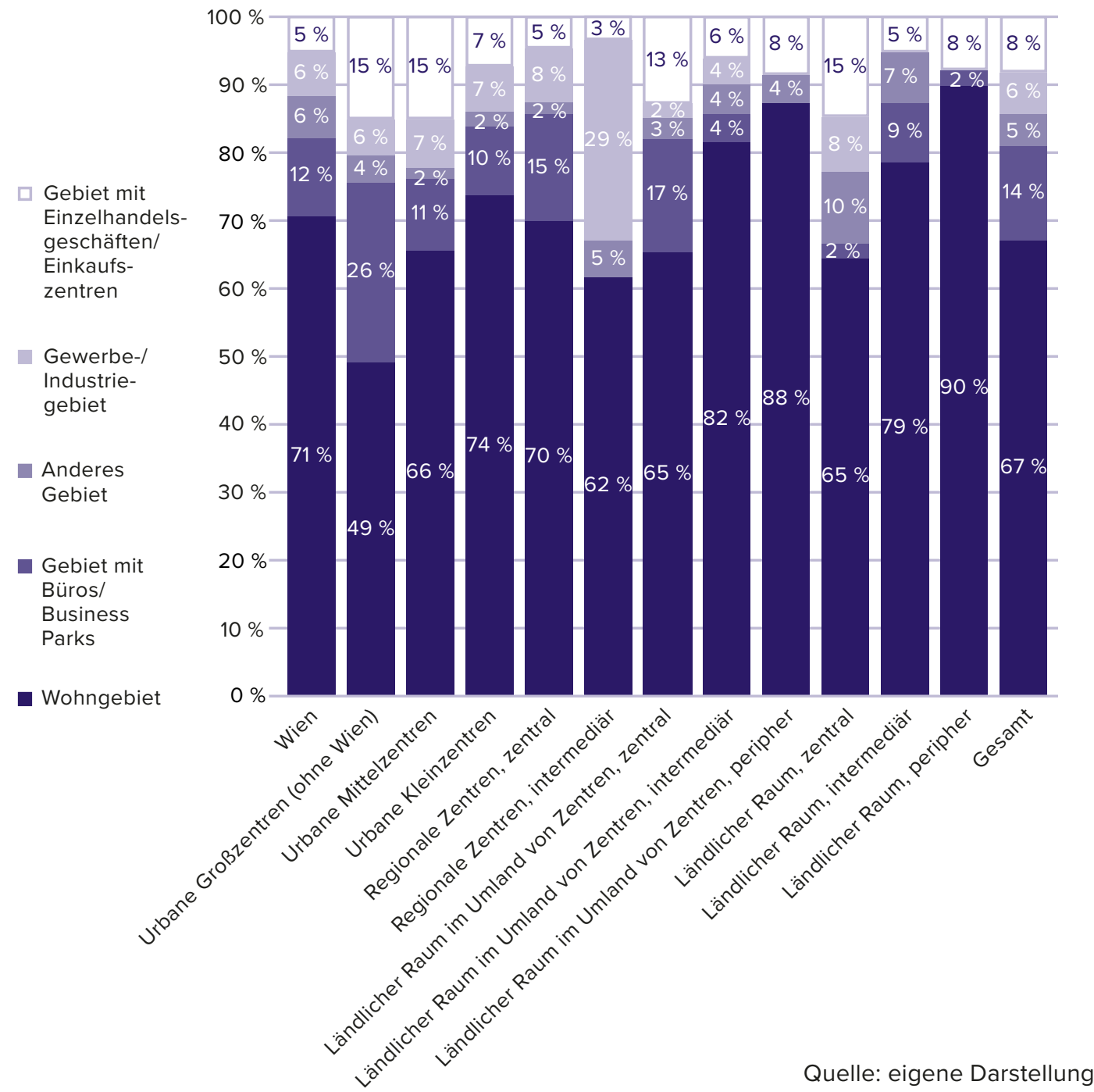




\section{Lage der Betriebe nach Entfernung zum Ortszentrum}

Hinsichtlich der Lage der Pkw-assoziierten Betriebe nach der Entfernung zum nächsten Ortszentrum zeigt sich, dass diese durchschnittlich 1,3 Kilometer vom nächsten Ortszentrum entfernt liegen (Abb. 16). Vor allem in peripheren Bereichen liegen die Betriebe relativ nahe zum nächsten Ortszentrum, insbesondere für die Kategorie "Ländlicher Raum, peripher" (695 m) und "Ländlicher Raum im Umland von Zentren, peripher" (861 m). Besonders in urbanen Mittelzentren $(1.441 \mathrm{~m})$ und urbanen Kleinzentren $(1.496 \mathrm{~m})$ liegen die Betriebe - vermutlich aufgrund der Siedlungsgröße - hingegen in deutlich weiterer Entfernung zum Ortszentrum.

Abbildung 16: Lage der Pkw-assoziierten Betriebe nach Entfernung zum Ortszentrum und UrbanRural-Typologie

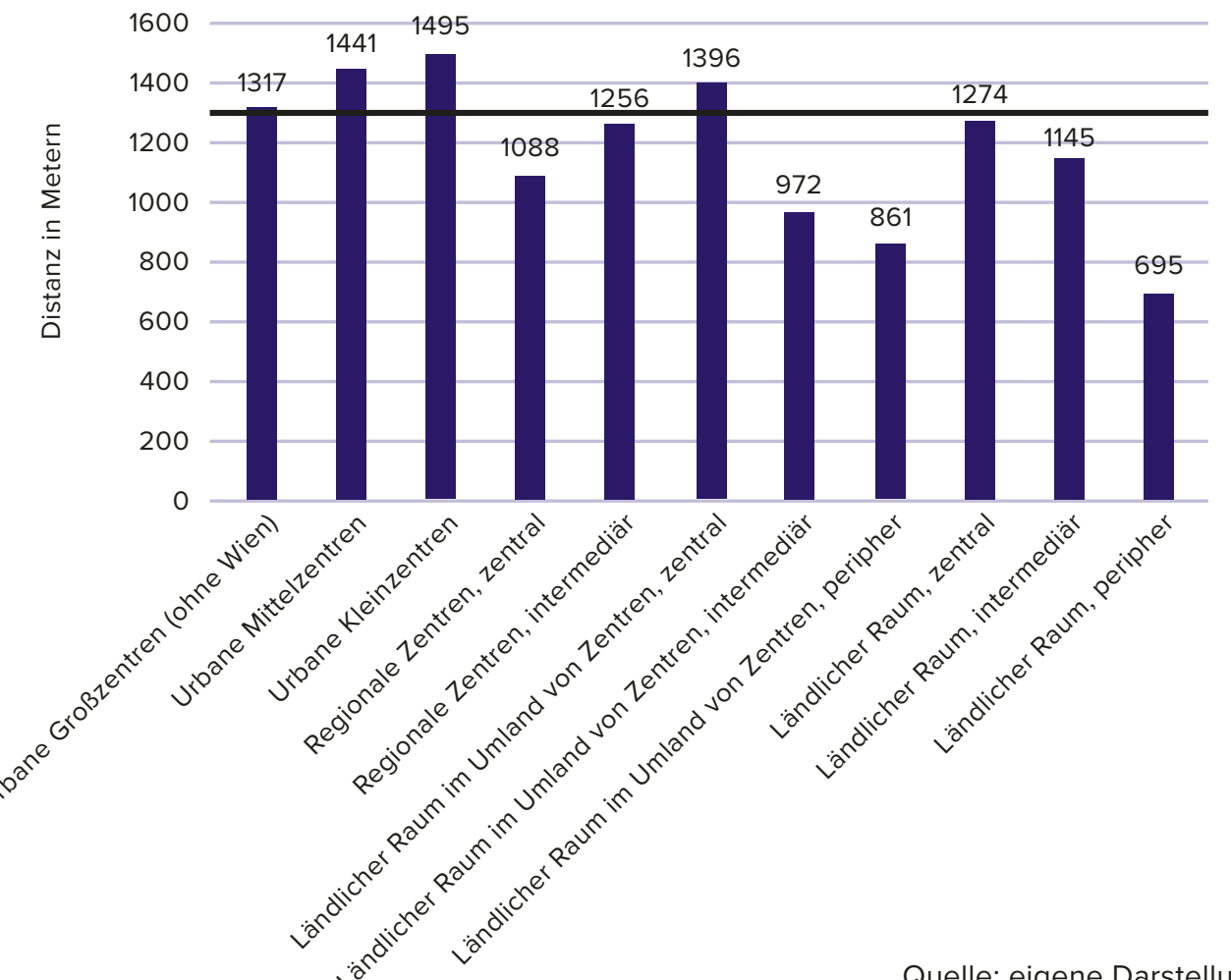

Quelle: eigene Darstellung

\subsection{VERTIEFTE UNTERSUCHUNG IN EINER BEISPIELGEMEINDE}

Für die vertiefte Untersuchung wurde auf Grundlage der Untersuchungsergebnisse auf Stadtregionsebene der 23. Wiener Gemeindebezirk Liesing als Beispielgemeinde gewählt. Die Analyse hinsichtlich der Anzahl an Pkw-assoziierten Betrieben und des Anteils dieser Betriebe an allen Betrieben bzw. Einrichtungen konnte aufzeigen, dass Liesing eines jener Gebiete ist, die sehr stark durch Pkw-assoziierte Betriebe geprägt ist. Darüber hinaus begründet sich die Relevanz von Liesing für diese Studie in der verkehrlich-räumlichen Ausgangslage: Am Stadtrand Wiens gelegen, ist Liesing sowohl an die U-Bahn (Linie U6) und die S-Bahn angebunden. Die hochrangigen Verkehrsachsen der Südbahn und der Autobahnen A2 (E59) und A21 (E60) führen durch das Gemeindegebiet. Die Gemeinde Liesing hat den höchsten Anteil an Autofahrten am Modal Split und ebenso die höchste Motorisierung aller Wiener Gemeindebezirke (ÖIR 2015). Die hohe Autoaffinität in Liesing wird mit den schlechten Anschlussverbindungen zum hochrangigen ÖPNV in Verbindung gebracht. Liesing ist ein stark wachsender Bezirk, mit einer hohen Nachfrage sowohl nach Wohnungseigentum als auch Mietwohnungen (Lottes 2020). Die Lage am Stadtrand und den Verkehrsachsen macht Liesing zusätzlich zu einem attraktiven Unternehmensstandort. 
Diese Gemengelage könnte Liesing zu einem hochrelevanten Zielgebiet für automatisierte Mobilitätsdienstleistungen machen (vgl. Beitrag 14 von Allmeier et al. in diesem Band). Auf ausgewählten Routen könnten attraktive Services in Ergänzung zum bestehenden ÖPNV möglich werden. Die in den letzten Jahren anhaltend dynamische Entwicklung der Gemeinde führt dazu, dass Themen der Innenentwicklung zusehends an Relevanz gewinnen. Aktuell wird ein Mangel an wohnungsnahen qualitativen Freiräumen als die zentrale Herausforderung beschrieben. Zusätzlich werden die Entwicklung eines feinmaschigeren ÖPNV-Netzes und die Entwicklung bzw. das Stärken von lokalen Stadtteilzentren gefordert und bereits gefördert (Magistratsabteilung 21 Stadtteilplanung und Flächennutzung 2015).

Die Pkw-assoziierten Konversionsflächen könnten sich bezüglich bestehender Ziele als wertvoll erweisen. Firmen aller Bereiche konzentrieren sich entlang der stark befahrenen Straßen wie etwa der Triester Straße, der Breitenfurter Straße oder der Ketzergasse (Abb. 17). Diese Straßen, die aufgrund ihrer hohen verkehrlichen Belastungen nicht als Transformationsräume gesehen wurden, könnten durch die hohen Flächenpotentiale grundlegend neu ausgerichtet werden.

Auch in den Industrie- und Gewerbegebieten, die sich südöstlich und weiter östlich des historischen Zentrums befinden, sind Cluster von Potentialflächen zu finden. Das Ziel, diese großen Gebiete besser städtebaulich zu integrieren, könnte so verfolgt werden. Über das gesamte Gemeindegebiet zeigen sich Potentiale, bestehende Grünräume im Sinne eines „Grünen Gerüsts“ (vgl. Magistratsabteilung 21 Stadtteilplanung und Flächennutzung 2015) zu vernetzen, Mobilitätsknoten zu entwickeln und Stadtteilzentren zu fördern.

Abbildung 17: Pkw-assoziierte Flächen in Liesing (23. Wiener Gemeindebezirk)

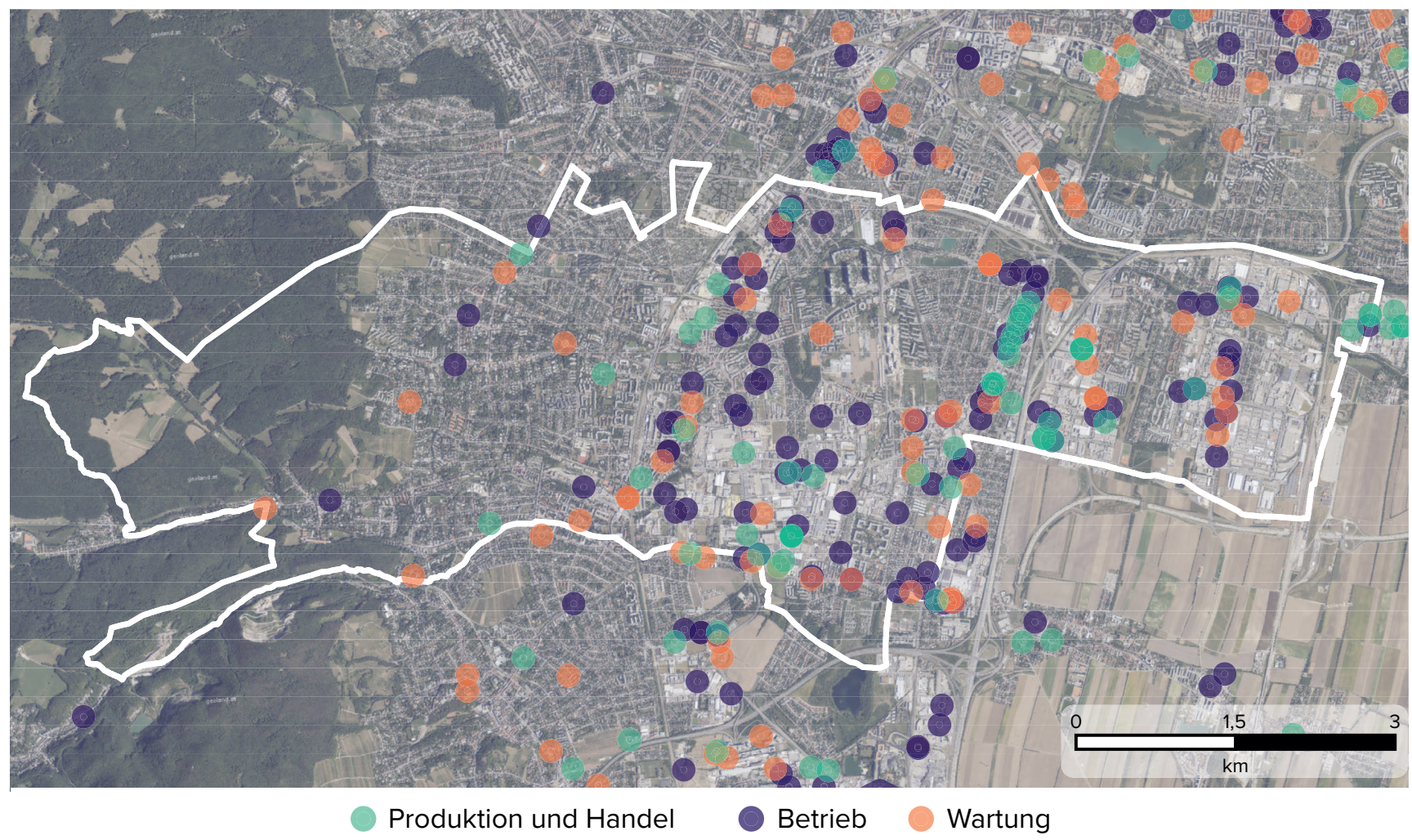

Quelle: eigene Darstellung auf Grundlage von basemap.at 


\section{CONCLUSIO UND DISKUSSION}

In seinem 1958 erschienenen und später überarbeiteten Text „The Highway and the City” warf Lewis Mumford seinen mit der Verkehrsplanung betrauten Kolleglnnen eine Geschichtsvergessenheit vor, die aus seiner Sicht desaströse städtebauliche und soziale Folgen für die Städte seiner Zeit hatte. ${ }^{2}$ Wie zu Zeiten des Eisenbahnbaus werden nach Mumford erneut Schneisen in die Städte geschlagen und wieder großzügig Flächen von allerlei Verkehrsbauten konsumiert (Mumford 1968). Das die Warnung Mumfords wenig Beachtung fand, zeigt vor allem die städtebauliche Gestaltung nordamerikanischer, aber auch europäischer Städte. Heute sind die Schneisen geschlagen und Pkw-assoziierte Flächen global über die Siedlungsstruktur verteilt. Am Beginn des Zeitalters automatisierter Mobilitätsservices stehen diese Flächen zur Debatte. Es bleibt zu hoffen, dass im Fall des anstehenden Wandels des Mobilitätssystems, Mumfords Ruf nach Geschichtskenntnis gehört wird. Die Gefahren bleiben im Falle von automatisierten Fahrzeugen die gleichen.

Dass die unüberlegte Flächenzuteilung zu dem jeweils neuen Verkehrsträger auch Chancen einer zukünftigen Stadtentwicklung bietet, zeigen die Vergangenheit und aktuelle Quartiersentwicklungsprojekte zum Beispiel an Güterbahnhöfen. Diese Flächen bildeten wertvolle Elemente der Quartiers- oder Stadtteilentwicklung, auf denen innerhalb der Siedlungsgrenzen neuer Wohn-, Arbeits- und Lebensraum geschaffen werden konnte. In den wachsenden Städten Europas ist diese Ressource nun erschöpft. Die neuen Quartiere sind gebaut bzw. verwertet. Während das Streckennetz der Eisenbahngesellschaften Europas schrumpfte, wuchsen ihre Immobilienresorts.

Die mit dieser Studie vorliegenden Ergebnisse könnten einen Beitrag dazu leisten, Mumfords Ruf qualifiziert umzusetzen. Angesichts bestehender Stadtentwicklungsziele europäischer Städte, Regionen und Kommunen, bei denen es zum einen um das Schaffen von lebenswerten urbanen Räumen und zum anderen um einen nachweisbaren Beitrag zu endlich festgelegten Klimazielen geht, könnten während der Transformation des Verkehrssystems wertvolle Entwicklungsflächen anfallen. Um weitere Bodenversiegelung (und dadurch induzierten Verkehr) zu vermeiden, spielt Innenentwicklung, verstanden als die „Erhöhung von Einheiten zum Wohnen und Arbeiten innerhalb des eingezonten und weitestgehend überbauten Gebiets bei gleichzeitiger Aufwertung des öffentlichen Raums und Anpassung der Infrastrukturen“ (Grams 2019), eine Schlüsselrolle. Die Entwicklung des Bestandes stellt allerdings bislang eine Herausforderung dar, der mit etablierten Mitteln der Planung nur schwer beizukommen ist. Flächen im Bestand zu sichern und differenzierten Nutzungen zuzuführen, d. h. auch einmal nicht zu bebauen, wird heute in der Planungspraxis nur selten erreicht.

Es ist davon auszugehen, dass die hier analysierten Flächenpotentiale, die durch einen systematischen Wandel des Verkehrssystems - vom Besitz hin zur Dienstleistung - nie vollumfänglich realisiert werden, über Jahrzehnte jedoch teilweise und Stück für Stück umgenutzt werden können. Die Untersuchung der FUA Wien und der Beispielgemeinde Liesing zeigt, dass durch differenziertes Vorgehen bestehende Entwicklungsziele durchaus erreicht werden können.

Quartiersentwicklung heute passiert realistisch betrachtet dort, wo es die Eigentümerstruktur zulässt, wo ein großer Eigentümer, etwa eine Eisenbahngesellschaft, zusammenhängende Flächen entwickeln will. Die Planungsmechanismen, die in der Vergangenheit geschaffen wurden,

2 „[They] lack both historic insight and social memory [and] accordingly, [...] have been repeating, with the audacity of confident ignorance, all the mistakes in urban planning committed by their predecessors who designed our railroads“" (Mumford 1968, 94). 
werden in Zukunft nicht mehr greifen. Die Ergebnisse dieser Analyse legen nahe, dass das Automobilzeitalter, selbst an seinem möglichen Ende, eine letzte Inversion bestehender Prinzipien verursachen könnte: Womit geplant werden muss, sind dispers über die Siedlungsstruktur verteilte Flächen mit einer ebenso dispersen Eigentümerstruktur. Es wird im Rahmen dieser Untersuchung jedoch deutlich, dass sich diese Flächen insbesondere in kleineren, ländlich geprägten Städten überwiegend in Wohngebieten befinden und diese Flächen vor allem in den regionalen Zentren und in ländlichen Bereichen eine überwiegende Nähe zum Ortszentrum aufweisen. Das Ausmaß und die Verteilung der Flächen in der Siedlungsstruktur, in tendenziell autoaffinen Lagen entlang von Hauptstraßen oder Autobahnen (dies wurde insbesondere im Rahmen der vertieften Untersuchung deutlich), zeigen somit Möglichkeiten einer kleinteiligen Akzentuierung, wie es einem aktuellen Planungsverständnis der Innenentwicklung entspricht.

Fahrdienstleister, aber auch kommunale oder regionale Verkehrsbetriebe könnten diese Flächen teilweise nutzen; die Analyse bestehender Standorte legt dies jedenfalls nahe. Es ist bekannt, dass die Marktführer hierfür hunderte Millionen Euro bereitgestellt haben (Hawkins 2018, Rapier/Wolverton 2019). Die neue Systematik automatisierter Mobilitätsservices dürfte allerdings gegenüber dem Individualverkehr zu einer erneuten Konzentration an Standorten führen, die auch erhebliche Folgen für lokal erbrachte Wertschöpfungen nach sich ziehen könnte.

Der in den bisher durchgeführten Studien liegende Fokus auf Parkplatzflächen zu automatisierten und vernetzten Fahrzeugen hat unter anderem dazu geführt, dass die wirtschaftliche Bedeutung des Wandels hin zu einem automatisierten, vernetzten und serviceorientierten Verkehrssystem lange nicht greifbar war (Clements/Kockelman 2017, Mitteregger et al. 2019): Mit dem Individualverkehr würde ein bedeutender Wirtschaftszweig mit lokaler und regionaler Wertschöpfung schwinden. Setzt sich die Logik der Sharing Economy vermehrt durch, entstehen an dessen Stelle prekäre Arbeitsplätze, von denen angenommen werden darf, dass sich der Druck auf diese mit vorschreitender Automatisierung im Verkehrssektor weiter erhöht.

Der Schlüssel zu einer Transformation, die lebenswerte Räume herstellt und einen Beitrag zur Bewältigung der globalen Klimakrise leistet, könnte darin liegen, den Straßenraum neu zu denken (Mitteregger et al. 2020). Gerade der Blick auf die ausgewählte Beispielgemeinde Liesing, die von der Triester Straße, einer der zentralen Pendlerachsen des Individualverkehrs, durchkreuzt wird, zeigt hier hochrelevante Potentiale. Der Cluster von Pkw-assoziierten Betrieben legt Gestaltungspotentiale offen, die nicht ungenutzt bleiben sollten. Tritt der Wandel wie prognostiziert ein, wäre dies ein kapitaler Fehler - und Mumford hätte schon wieder recht behalten.

\section{LITERATUR}

Alessandrini, A., A. Campagna, P. Delle Site, F. Filippi und L. Persia 2015. „Automated vehicles and the rethinking of mobility and cities“, in Transportation Research Procedia 5, 145-160.

Anair, D., J. Martin, M. C. de Moura und J. Goldman 2020. Ride-Hailing's Climate Risks: Steering a Growing Industry Toward a Clean Transportation Future. Cambridge, MA: Union of Concerned Scientists. https://tinyurl.com/tz5gwtt (15.4.2020).

Banham, R. 1971. Los Angeles: The architecture of four ecologies. Los Angeles: University of California Press.

Bauer, C. 2011. 7 Tools zur Innenentwicklung: Die Metron Dichtebox: der Siedlungsraum der Schweiz soll begrenzt und die Entwicklung nach Innen gelenkt werden. Brugg: Metron. https://bit. ly/38sZRzj (4.5.2020).

Beiker, S. 2015. Einfühungsszenarien für höhergradig automatisierte Straßenfahrzeuge, in Autonomes Fahren. Technische, rechtliche und gesellschaftliche Aspekte, hg. v. M. Maurer, J. C. Gerdes, B. Lenz und H. Winner. Berlin/Heidelberg: Springer Vieweg, 197-218. 
Bellet, C. 2009. „The introduction of the high speed rail and urban restructuring: the case of Spain“, in City Futures 9, 4-6.

Bertolini, L. 2017. Planning the Mobile Metropolis: Transport for People, Places and the Planet. London: Palgrave/Red Grove Press.

Bertolini, L., C. Curtis und J. Renne 2012. „Station area projects in Europe and beyond: Towards transit oriented development?", in Built Environment (38) 1, 31-50.

Bertolini, L., und T. Spit 2005. Cities on rails: The redevelopment of railway stations and their surroundings. London: Routledge.

Bormann, R., P. Fink, H. Holzapfel, S. Rammler, T. Sauter-Servaes, H. Tiemann, T. Waschke und B. Weirauch 2018. „Die Zukunft der deutschen Automobilindustrie: Transformation by Disaster oder by Design?“, WISO Diskurs 03/2018. Bonn: Friedrich-Ebert-Stiftung.

Bourdieu, P. 1987. Die feinen Unterschiede. Frankfurt am Main: Suhrkamp.

Braham, W. W., und J. A. Hale 2007. Rethinking Technology: A Reader in Architectural Theory. London: Routledge.

Bruinsma, F., E. Pels, H. Priemus, P. Rietveld und B. van Wee 2008. Railway development: Impact on urban dynamics. Amsterdam: Physica.

Burghart, W., und G. Hertenberger 2018. Österreichs gefährdetes Kulturerbe: Vom Umgang mit dem Denkmalschutz: 70 Fallbeispiele. Wien: Schreybgasse.

Chan, N. D., und S. A. Shaheen 2012. Ridesharing in North America: Past, present, and future. Transport Reviews (32) 1, 93-112.

Charpentier, M. 2020. „Lyft Unveils ,One-Stop-Shop“ Service Center For Drivers In Austin“, KUT 90.5. Austin's NPR Station, 14.1.2020. https://tinyurl.com/qs2xrp2 (4.5.2020).

Chaum, M. 2019. „Uber Video Keynote“, Vortrag bei der Schweizer Mobilitätsarena, 16.9.2019.

Clements, L. M., und K. M. Kockelman 2017. „Economic Effects of Automated Vehicles“, in Transportation Research Record (2606) 1, 106-114.

Cohen, M. A., N. Agrawal und V. Agrawal 2006. „Winning in the aftermarket“, in Harvard Business Review (84) 5, 129-146.

Cresswell, T. 2006. On the Move: Mobility in the Modern Western World. New York: Routledge.

Eckl-Dorna, W. 2020. „So treibt der Dieselskandal Autohäuser in den Ruin“, Der Spiegel, 20.2.2020. https://tinyurl.com/qvpk9fw (15.4.2020).

Edelson, Z. 2017. „Port Authority Bus Terminal to get total reset and other breaking news from annual RPA conference“, The Architect's Newspaper, 21.4.2017. https://tinyurl.com/sx6dvks (15.4.2020).

Epting, S. 2019. „Automated vehicles and transportation justice“, in Philosophy \& Technology (32) 3, 389-403.

Frangoul, A. 2018. „How remote control centers are changing the way mining operations are carried out“, CNBC, 4.7.2018. https://tinyurl.com/r2luk4m (15.4.2020).

Freeman, C., und C. Perez 1998. Structural crises of adjustment: business cycles and technical change and economic theory. London: Pinter.

Geels, F. W., und J. Schot 2010. „The dynamics of transitions: a socio-technical perspective“, in Transitions to Sustainable Development: New Directions in the Study of Long Term Transformative Change, hg. v. J. Grin, J. Rotmans und J. Schot. London: Routledge, 11-104.

Görgl, P., J. Eder, E. Gruber und H. Fassmann 2017. „Monitoring der Siedlungsentwicklung in der Stadtregion+Strategien zur räumlichen Entwicklung der Ostregion”, Studie im Auftrag der Planungsgemeinschaft Ost. https://tinyurl.com/trqqwdl (16.4.2020).

Grams, A. 2017. „Spielräume für Dichte. Der Innenentwicklungskompass als problemorientierte Methode für Verdichtung in kleinen und mittleren Gemeinden“, IRL-Bericht 8. Zürich: vdf Hochschulverlag.

Greenblatt, J. B., und S. Shaheen 2015. „Automated Vehicles, On-Demand Mobility, and Environmental Impacts“, in Current Sustainable/Renewable Energy Reports (2) 3, 74-81.

Grübler, A. 1990. The rise and fall of infrastructures. Heidelberg: Physica.

Grübler, A. 1992. „Technology and Global Change: Land Use, Past and Present“, IIASA Working Paper WP-92-002, Laxenburg.

Hagemann, I. 2016. „Das gegenhegemoniale Moment der Demokratie. Gegenhegemoniale Projekte und demokratische Demokratie am Fallbeispiel der grünen Bewegung“. Universität Duisburg-Essen. 
Hall, P. G., und K. Pain (Hrsg.) 2006. The polycentric metropolis: learning from mega-city regions in Europe. London: Routledge.

Hawkings, A. J. 2018. „Lyft will spend \$100 million on new driver support centers“, The Verge, 23.5.2018. https://tinyurl.com/ycb94tw2 (16.4.2020).

Headrick, D. R. 1994. „Technological Change“, in The Earth as Transformed by Human Action, hg. v. R. Turner. Cambridge: Cambridge University Press, 55-68.

Heinrichs, D. 2016. „Autonomous driving and urban land use“, in Autonomous Driving. Technische, rechtliche und gesellschaftliche Aspekte, hg. v. M. Maurer, J. C. Gerdes, B. Lenz und H. Winner. Berlin/Heidelberg: Springer Vieweg, 213-231.

Heller, N. 2017. „Is the Gig Economy Working?“, The New Yorker, 15.5.2017. https://tinyurl.com/utlvmct (15.4.2020).

Hu, W. 2017. „As Uber Woos More Drivers, Taxis Hit Back“, The New York Times, 28.3.2017. https:// tinyurl.com/vv8cqcm (15.4.2020).

Jolliet, O., und P. Crettaz 1996. „Critical surface-time 95. A life cycle impact assessment methodology including fate and exposure“, EPFL -École polytechnique fédérale de Lausanne, 1996.

Kucharczyk, S. 2017. „How will maintenance change with the autonomous vehicle?", ReadWrite, 18.4.2017. https://tinyurl.com/td6s2hf (15.4.2020).

Kuhn, T. S. 1967. The structure of scientific revolutions. Chicago: The University of Chicago Press.

Leberstein, S. 2016. „Uber's car leasing program turns its drivers into modern-day sharecroppers“, Quartz, 6.6.2016. https://tinyurl.com/yx7oecf5 (16.4.2020).

Leiner, C., und T. Adler 2019. „Transportation Network Companies (TNCs): Impacts to Airport Revenues and Operations - Reference Guide“, ACRP Research Report 215.

Lenz, B., und E. Fraedrich 2015. „Neue Mobilitätskonzepte und autonomes Fahren: Potenziale der Veränderung “, in Autonomes Fahren. Technische, rechtliche und gesellschaftliche Aspekte, hg. v. M. Maurer, J. C. Gerdes, B. Lenz und H. Winner. Berlin/Heidelberg: Springer Vieweg, 175-195.

Lottes, G. J. 2020. „Der Wohnungsmarkt in Wien. Ein Marktbericht von Raiffeisen“, Raiffeisenlandesbank Niederösterreich-Wien. https://tinyurl.com/tn55auo (16.4.2020).

Magistratsabteilung 21 (Stadtteilplanung und Flächennutzung) 2015. „Perspektive Liesing. Ein Entwicklungskonzept für einen Stadtteil im Wachsen“, Projektzeitung \#2, 2.1.2015. https://tinyurl.com/ tnjtguc (16.4.2020).

Manderscheid, K. 2014. „The Movement Problem, the Car and Future Mobility Regimes: Automobility as Dispositif and Mode of Regulation“, in Mobilities (9) 4, 604-626.

Marshall, S. 2005. Street \& Patterns. London: Spon Press.

McKinsey 2018. „Ready for inspection - The Automotive Aftermarket in 2030“. https://tinyurl.com/ ssb7ksk (15.4.2020).

McLuhan, M. 2003, Understanding Media: The extension of man. Corte Madera: Ginko Press.

Mitteregger, M., A. Soteropoulos, J. Bröthaler und F. Dorner 2019. „Shared, Automated, Electric: the Fiscal Effects of the ,Holy Trinity“", Proceedings of the 24. REAL CORP, International Conference on Urban Planning, Regional Development and Information Society. Karlsruhe.

Mitteregger, M., E. M. Bruck, A. Soteropoulos, A. Stickler, M. Berger, J. S. Dangschat, R. Scheuvens und I. Banerjee 2020. AVENUE21. Automatisierter und vernetzter Verkehr: Entwicklungen des urbanen Europa. Berlin: Springer Vieweg.

Mumford, L. 1968. „The highway and the city“, in The Urban Prospect, hg. v. L. Mumford L. New York: Harcourt, Brace \& World, 92-107.

OECD 2020. „Functional urban areas by country“. https://tinyurl.com/tawbkmz (15.4.2020).

OpenStreetMap Wiki 2020. „DE:Key:landuse“. https://tinyurl.com/vo6prk9 (16.4.2020).

Ongweso Jr., E., und J. Koebler 2019. „Uber Office Has Nice Port-a-Potties for ,Employees Only, Inferior Ones for Drivers“, VICE, 5.12.2019. https://tinyurl.com/ufc2jxx (15.4.2020).

Pevsner, N. 1953. The Buildings of England: Derbyshire. Harmondsworth: Penguin.

Ramm, F. 2019. „OpenStreetMap Data in Layered GIS Format“. https://tinyurl.com/yyuksIna (16.4.2020).

Rapier, G., und T. Wolverton 2019. „Uber lost $\$ 5.2$ billion in 3 months. Here's where all that money went“, Business Insiderm 9.8.2019. https://tinyurl.com/yxxsm2qf (16.4.2020).

Rogers, E. M. 2003. Diffusion of innovations. New York: Free Press. 
Schmitz, S. 2001. Revolutionen der Erreichbarkeit. Gesellschaft, Raum und Verkehr im Wandel (Stadtforschung aktuell, Band 83). Wiesbaden: VS Verlag für Sozialwissenschaften.

SFCTA (San Francisco Country Transportation Authority) 2018. „TNCs \& Congestion. Final Report.“ https://tinyurl.com/y6w76ta6 (15.4.2020).

Shaheen, S., und A. Cohen 2019. „Shared ride services in North America: definitions, impacts, and the future of pooling“, in Transport Reviews (39) 4, 427-442.

Sheikh, N. 2018. „Applying a Hybrid Network Approach to Deployment of Self-Driving Mobility Services“, Automated Vehicle Symposium. https://tinyurl.com/uhwtb3k (15.4.2020).

Sieverts, T. 1998. Zwischenstadt: Zwischen Ort und Welt, Raum und Zeit, Stadt und Land, Bauwelt Fundamente 118. Wiesbaden: Vieweg+Teubner.

Sommer, C. 2018. „Neue Angebote für den ländlichen Raum“, Vortrag beim Symposium „ZENTRALITÄTEN 4.0 - Mittelzentren im Zeitalter der Digitalisierung“, 22.11.2018, Kassel.

Soteropoulos, A., M. Berger und F. Ciari 2019. „Impacts of automated vehicles in travel behaviour and land use: An international review of modelling studies“, in Transport Reviews (39) 1, 29-49.

Soteropoulos, A., M. Mitteregger, M. Berger und J. Zwirchmayr 2020. „Automated drivability: Toward an assessment of the spatial deployment of level 4 automated vehicles“, in Transportation Research Part A: Policy and Practice 136, 64-84.

Spielmann, M., und R. Scholz 2005. „Life Cycle Inventories of Transport Services: Background Data for Freight Transport", in The International Journal of Life Cycle Assessment (10) 1, 85-94.

Splend 2019. „We have a new Member Support Centre in London“, Pressemitteilung, 17.4.2019. https:// tinyurl.com/s3uwy97 (15.4.2020).

Statistik Austria 2017. „Urban-Rural-Typologie von Statistik Austria“. https://tinyurl.com/rs5qj9s (16.4.2020).

Stead, D., und B. Vaddadi 2019. „Automated vehicles and how they may affect urban form: A review of recent scenario studies“, in Cities 92, 125-133.

Steen, B., und S.-O. Ryding 1993. The EPS enviro-accounting method. An application of environmental accounting for evaluation and valuation of environmental impact in product design. APR: Stockholm.

STRIA 2019. Roadmap on Connected and Automated Transport: Road, Rail and Waterborne. Brüssel: Europäische Kommission.

Tucker, C. 2019. „Uber-rival Bolt opens new hub in Chiswick, London, surging to 1.5 million London passengers in 6 months“, EU-Startups, 25.12.2019. https://tinyurl.com/sbay9ul (15.4.2020).

Vlasic, B. \& Isaac, M. 2016. „Uber Aims for an Edge in the Race for a Self-DrivingFuture“, The New York Times, 18.8.2016. https://tinyurl.com/w928p8a (16.4.2020).

Yankelevich, A., R. V. Rikard, T. Kadylak, M. J. Hall, E. A. Mack, J. P. Verboncoeur und S. R. Cotton 2018. „Preparing the Workforce for Automated Vehicles“. https://bit.ly/3qrzXC8 (15.4.2020).

Zwick, A. 2018. „Welcome to the Gig Economy: neoliberal industrial relations and the case of Uber", in GeoJournal (83) 4, 679-691.

Open Access Dieser Beitrag wird unter der Creative Commons Namensnennung 4.0 International Lizenz (http://creativecommons.org/licenses/by/4.0/deed.de) veröffentlicht, welche die Nutzung, Vervielfältigung, Bearbeitung, Verbreitung und Wiedergabe in jeglichem Medium und Format erlaubt, sofern Sie den/die ursprünglichen Autor(en) und die Quelle ordnungsgemäß nennen, einen Link zur Creative Commons Lizenz beifügen und angeben, ob Änderungen vorgenommen wurden.

Die in diesem Beitrag enthaltenen Bilder und sonstiges Drittmaterial unterliegen ebenfalls der genannten Creative Commons Lizenz, sofern sich aus der Abbildungslegende nichts anderes ergibt. Sofern das betreffende Material nicht unter der genannten Creative Commons Lizenz steht und die betreffende Handlung nicht nach gesetzlichen Vorschriften erlaubt ist, ist für die oben aufgeführten Weiterverwendungen des Materials die Einwilligung des jeweiligen Rechteinhabers einzuholen.

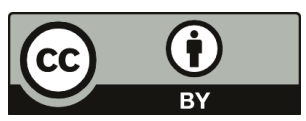

